\title{
14. Geldzahlungen, Sachzuwendungen und Kostenerstattung im freiwilligen Engagement
}

\author{
Laura Romeu Gordo \& Claudia Vogel
}

\section{Kernaussagen}

Geldzahlungen und Sachzuwendungen im freiwilligen Engagement sind selten. Der größte Anteil der Engagierten erhält keine Geldzahlung. Die Engagierten, die Geld erhalten (9,9 Prozent), bekommen zudem in der Regel niedrige Beträge. Der Anteil der Engagierten, die Sachzuwendungen erhalten, ist ebenfalls nicht hoch, nur 14,6 Prozent der Engagierten erhalten eine solche. Allerdings erhalten Schülerinnen und Schüler überdurchschnittlich häufig Sachzuwendungen.

Die Unterschiede zwischen den Engagementbereichen sind bei den Geld- und Sachleistungen stark ausgeprägt. Die Wahrscheinlichkeit, Geldzahlungen und Sachzuwendungen für freiwillige Tätigkeiten zu erhalten, hängt vom gesellschaftlichen Bereich ab (Engagierte im Bereich Justiz und Kriminalitätsprobleme erhalten am häufigsten Geld, Engagierte im Bereich Jugendarbeit erhalten am häufigsten Sachzuwendungen). Meist werden die Leistungen nicht kombiniert, das heißt, die freiwillig Engagierten erhalten in der Regel entweder Geld oder Sachzuwendungen.

Schülerinnen und Schüler erhalten im Zeitvergleich mehr Sachzuwendungen. Der Anteil der Engagierten, die Geldzahlungen erhalten, hat im Vergleich zum Jahr 1999 abgenommen. Im Gegensatz dazu hat sich der Anteil der Personen, die Sachzuwendungen erhalten, mehr als verdoppelt. Dieser Anstieg geht zu großen Teilen auf die Steigerung bei den Schülerinnen und Schülern zurück.

Freiwilliges Engagement verursacht häufig Kosten für die Engagierten. Knapp die Hälfte der engagierten Personen berichtet über entstandene Kosten bei ihrem freiwilligen Engagement. Kosten für Engagement entstehen häufiger in höheren Einkommensgruppen. Für mehr als die Hälfte der Engagierten mit einem monatlichen Haushaltseinkommen von über 4.000 Euro entstehen Kosten. Bei Engagierten, deren Haushaltseinkommen 1.000 Euro oder weniger beträgt, berichtet jeder Dritte von entstandenen Kosten.

Die durch das Engagement entstehenden Kosten werden selten erstattet. Nicht einmal jede beziehungsweise jeder fünfte Engagierte hat die Möglichkeit, sich diese Kosten erstatten zu lassen, und auch wenn die Möglichkeit besteht, nutzen viele Engagierte diese nicht.

\subsection{Einleitung}

In der letzten Dekade hat sich der Diskurs um die Monetarisierung des freiwilligen Engagements verstärkt. Den Anstoß lieferte hauptsächlich die Diskussion über die Praxis großer Organisatio- nen, Geldzahlungen wie Stundenlöhne oder Taschengeld für Ehrenamtliche einzuführen, oder Übungsleiterpauschalen $\mathrm{zu}$ bezahlen wie im Bereich Sport (Schumacher 2015: 44ff). Es gibt

(C) Der/die Autor(en) 2017

J. Simonson et al. (Hrsg.), Freiwilliges Engagement in

Deutschland, Empirische Studien zum bürgerschaftlichen

Engagement, DOI 10.1007/978-3-658-12644-5_15 
zahlreiche Formen von materiellen Tauschwerten im freiwilligen Engagement (Zentrum für Zivilgesellschaftliche Entwicklung 2009). Es kann unterschieden werden nach direkten Geldzahlungen (Auslagenersatz, pauschale Gratifikation, Vergütungen, Entschädigung von Verdienstausfall), geldwerten Leistungen (Sachzuwendungen, Bildungsmaßnahmen, private Mitnutzung technischer Infrastruktur), reduzierten Zahlungspflichten (Ermäßigungen, steuerrechtliche Privilegierung), Zeitkonten und Anwartschaften auf Rentenversicherungsbezüge. Im Freiwilligensurvey liegen Informationen zum einen $\mathrm{zu}$ Geldleistungen und zum anderen zu geldwerten Leistungen in Form von Sachzuwendungen vor. Der Begriff der Monetarisierung bezeichnet die „Praxis direkter oder indirekter Geldzahlungen im Rahmen von Tätigkeiten, die nach dem Selbstverständnis von Engagierten und Organisationen als freiwilliges, ehrenamtliches oder bürgerschaftliches Engagement gelten“ (Zentrum für Zivilgesellschaftliche Entwicklung 2009: 9). Zudem wird der Begriff auch verwendet, um einen Trend beziehungsweise den „Prozess der allgemeinen Zunahme von Tätigkeitsformen im Zwischenbereich von unbezahltem freiwilligem Engagement und regulärer Erwerbsarbeit" zu beschreiben (Zentrum für Zivilgesellschaftliche Entwicklung 2009: 9). Dieses prozesshafte Verständnis der Monetarisierung deutet bereits eine normative Wertung an: Bei Tätigkeiten, für welche die Engagierten Geld bekommen, kann es sich im strengen Sinne nicht um freiwilliges Engagement handeln.

In der wissenschaftlichen Literatur stellt sich die Diskussion um den Einfluss von Geldzahlungen auf die Motivation der Individuen ebenso kontrovers dar wie in der politischen Debatte. Der klassischen ökonomischen Literatur folgend, sollten Geldzahlungen tatsächlich einen Anstieg des Anteils freiwillig Engagierter verursachen, weil Einkommen grundsätzlich ein erstrebenswertes Gut ist. Diese Modelle betrachten aber nur die extrinsische, von außen wirkende Motivation der Personen und vernachlässigen die intrinsische Motivation der Engagierten. Per- sonen sind intrinsisch motiviert, eine Tätigkeit auszuüben, wenn sie die Tätigkeit nicht aufgrund einer zusätzlichen Belohnung ausüben, sondern allein aufgrund der Belohnung durch die Tätigkeit selbst - etwa das Gefühl, etwas Sinnvolles zu tun, gebraucht zu werden, helfen zu können oder seine Meinung vertreten zu dürfen. Wenn Geldzahlungen für freiwillige Tätigkeiten eingeführt werden, kann sich die Wahrnehmung der Engagierten bezüglich ihrer Tätigkeit ändern und dadurch die intrinsische Motivation verloren gehen. Dieser Effekt kann dazu führen, dass Geldzahlungen an Menschen dafür, dass sie genau das tun sollen, was sie bisher getan haben, einen Rückzug aus dem Engagement und somit einen niedrigeren Anteil Engagierter verursachen. Dieses Phänomen ist in der Literatur als, Motivation Crowding Effect' (Frey \& Jegen 2001) bekannt. Ein Beispiel findet sich in der Forschung zur Bereitschaft Blut zu spenden: Diese Bereitschaft sinkt bei Zahlung eines Honorars anstatt, wie eigentlich gewünscht, anzusteigen (Priller \& Schupp 2011; Schupp 2012; Grant 2012).

Obwohl diese Befunde gegen eine Empfehlung zur Monetarisierung als Anreizfunktion im freiwilligen Engagement zu sprechen scheinen, sollte zusätzlich auch die Höhe der geleisteten Geldzahlungen berücksichtigt werden. Finanzielle Leistungen und Sachzuwendungen sind auch eine von Engagierten gewünschte Form der Anerkennung für ihr Engagement (siehe Kapitel 20). Wenn jedoch mehr Geld ins Spiel kommt, verwischen die Grenzen zwischen Erwerbsarbeit und freiwilligem Engagement möglicherweise tatsächlich. Zahlungen, die über die durch das Engagement verursachten Kosten hinausgehen, können zu einer Einbindung der bezahlten Person in eine Hierarchie der Erwerbsarbeit führen. In einer solchen bleiben aber möglicherweise die Freiwilligkeit und die Eigenmotive der Engagierten unberücksichtigt (Olk \& Klein 2007). Darüber hinaus können auch die Freiheit des Handelns - wer zahlt, der bestimmt (Evers 2006) - und die Freiheit, auf Probleme hinzuweisen, verloren gehen (Jakob 2009). Vandamme sieht in der Selbstbestimmung ebenfalls einen grund- 
sätzlichen Unterschied zwischen Engagement und Erwerbsarbeit: „Monetarisierung bringt Anreiz und Anerkennung, aber auch Einschränkung der Selbstbestimmung. Wer bezahlt, bestellt. Und entlässt“ (2007: 2). Damit würden die Merkmale und Ziele der Arbeitswelt in die Welt des Engagements eindringen, was von Gegnerinnen und Gegnern der Monetarisierung abgelehnt wird (Evers 2007). Durch die Ausübung einer freiwilligen Tätigkeit wird jedoch kein Arbeitsverhältnis begründet (Armbrüster 2014). Ob empirisch tatsächlich eine zunehmende Tendenz zu Geldzahlungen im freiwilligen Engagement zu beobachten ist, wie bisher angenommen wird, betrachten wir für den Beobachtungszeitraum von fünfzehn Jahren (1999 bis 2014). Darüber hinaus untersuchen wir, ob es bezüglich der Geldzahlungen und Sachzuwendungen Unterschiede zwischen den gesellschaftlichen Engagementbereichen gibt. Sachzuwendungen umfassen Verschiedenes, sie können in Form der Nutzung von Räumen oder auch von Fahrzeugen erbracht werden, oder in Form von Gutscheinen oder Freitickets, es kann sich aber auch um Sachgeschenke oder Ausrüstungsgegenstände wie Bälle, Trikots oder Schutzwesten handeln, die in den verschiedenen Engagementbereichen unterschiedlich weit verbreitet sind.

In der Diskussion der zunehmenden Monetarisierung bei freiwilligen Tätigkeiten wird oft nicht zwischen Geldzahlungen und Kostenerstattung differenziert. Dabei ist diese Differenzierung sehr wichtig. Geldzahlungen bedeuten einen finanziellen Zugewinn für die Person, während es bei einer Kostenerstattung nur um den Ausgleich von Auslagen einer Person geht. Während finanzielle Gewinne eine Anreizfunktion haben können, spielt Kostenerstattung eine wichtige Rolle, um die finanzielle Barriere für freiwilliges Engagement zu reduzieren. Geldzahlungen für freiwillige Tätigkeiten sind oft als Aufwandsentschädigung für entstandene Kosten zu verstehen, da die Spende von Zeit - unentgeltliches Engagement - häufig mit dem Auslegen von Geld (z. B. der Übernahme von Fahrtkosten) in Verbindung steht (Olk \& Klein 2007). Laut Ebert besteht auch eine historische Tradition öffentlicher Ehrenämter (z. B. Bürgermeisteramt, Wahlhelferin oder -helfer), bei denen eine Person „zur Übernahme eines solchen Amtes sogar verpflichtet werden kann, dann aber auch einen Anspruch auf finanziellen Nachteilsausgleich hat" (2011: 284). Aufwandsentschädigungen werden also sowohl als Kosten- oder Auslagenersatz als auch als Entschädigung für einen geleisteten Zeitaufwand - zum Teil sogar als Kompensation von Verdienstausfällen im Beruf - eingesetzt (vgl. Ebert 2011). Über Aufwandsentschädigungen hinaus soll freiwilliges Engagement haftungs- und unfallrechtlich abgesichert werden. Die Idee dabei ist, dass die Engagierten etwa im Falle eines Unfalls nicht durch zusätzliche Kosten benachteiligt werden sollen (Jakob 2009).

Tatsächlich können die durch ein Engagement entstehenden Kosten eine Hürde darstellen, sich zu engagieren, insbesondere für Personen mit geringen Einkommen. Durch Aufwandsentschädigungen, die die entstehenden Kosten abdecken, können solche finanziellen Barrieren jedoch reduziert werden; am besten, indem einkommensarme Engagierte erst gar kein Geld vorstrecken müssen, aber auch, indem ihnen Kosten nachträglich erstattet werden. Durch Kostenerstattungen wird möglicherweise die soziale Ungleichheit im Zugang zu Engagement abgeschwächt. Sonst könnten es sich manche lediglich deshalb nicht leisten, sich zu engagieren, weil ihnen die Mittel fehlen, etwa Fahrtkosten oder sonstige Auslagen wie Materialkosten aufzubringen. Ein Abbau der Zugangshemmnisse wäre jedoch nur dann zu beobachten, wenn finanziell schlechter gestellte Engagierte häufiger eine Aufwandsentschädigung erhalten als finanziell besser gestellte. Erhalten hingegen diejenigen häufiger Kostenerstattungen, die besser gestellt sind, verschärfen diese Geldzahlungen potenziell die soziale Ungleichheit.

In diesem Kapitel untersuchen wir, wie sich Kosten und die Nutzung der Möglichkeit, Kosten erstatten zu lassen, zwischen Männern und Frauen sowie zwischen verschiedenen Einkommensund Altersgruppen unterscheiden. Wir vermuten 
Geschlechter-, Bildungs- und Einkommensunterschiede, weil die zur Verfügung stehenden Ressourcen in diesen Gruppen unterschiedlich verteilt sind, aber auch, weil sich die Personen in unterschiedlichen Bereichen engagieren und verschiedene Tätigkeiten unterschiedlich häufig Kosten verursachen. Darüber hinaus wird auch untersucht, in welchen Bereichen Kosten des freiwilligen Engagements zu welchen Anteilen vorkommen, sodass wir über höhere finanzielle Barrieren in diesen Bereichen sprechen können.

In diesem Kapitel soll folgenden Forschungsfragen nachgegangen werden:

1. Wie verbreitet sind Geldzahlungen und Sachzuwendungen im freiwilligen Engagement und wie unterscheidet sich der Erhalt zwischen Bevölkerungsgruppen?

\subsection{Datengrundlage}

Die in diesem Kapitel berichteten Befunde basieren auf gewichteten Analysen. Für die Gewichtung wurden in allen Erhebungsjahren dieselben Gewichtungsmerkmale berücksichtigt. Dabei handelt es sich um die Designgewichtung, die sich auf die Auswahlwahrscheinlichkeit einer Person bezieht (Haushaltsgröße, Zahl der Festnetznummern und, nur für das Jahr 2014, auch die Zahl der Mobilfunknummern) und Gewichtungsmerkmale, die Abweichungen der Stichprobenverteilung von der Grundgesamtheit in bestimmten Dimensionen ausgleichen (Bundesland, Gemeindegrößenklassen, Geschlecht, Altersgruppen). Von einer Hinzunahme weiterer Gewichtungsmerkmale wie etwa des Bildungsstandes haben wir abgesehen, um die Vergleichbarkeit mit den bisher veröffentlichten Berichten zu erhalten.
2. In welchen Engagementbereichen kommen Geldzahlungen oder Sachzuwendungen häufig, in welchen Bereichen selten vor?

3. Haben die Geldzahlungen und Sachzuwendungen für das freiwillige Engagement im Zeitvergleich zugenommen?

4. Wie häufig entstehen Kosten und wie verbreitet sind Kostenerstattungsmöglichkeiten im freiwilligen Engagement? Wie unterscheiden sich die Kosten und die Kostenerstattungsmöglichkeiten zwischen verschiedenen Bevölkerungsgruppen?

5. In welchen Engagementbereichen entstehen den Engagierten häufiger Kosten und in welchen Bereichen können sie sich diese häufiger erstatten lassen beziehungsweise in welchen Bereichen lassen sie sich diese häufiger erstatten?

Wir untersuchen sowohl Kosten und Kostenerstattungsmöglichkeiten im freiwilligen Engagement als auch Geldzahlungen und Sachzuwendungen für die Engagierten. Eine Inanspruchnahme der Kostenerstattung stellt keinen finanziellen Zugewinn dar, weil die Erstattung in der Regel lediglich in Höhe der tatsächlich entstandenen Kosten und meist gegen Nachweis erfolgt. Geldzahlungen und Sachzuwendungen stellen dagegen in der Regel einen Zugewinn für die Person dar. Im Falle von pauschalierten Aufwandsentschädigungen kann die Kostenerstattung vom Zugewinn jedoch nicht getrennt werden. Es könnte nämlich sein, dass die Kosten, die einer freiwillig engagierten Person durch ihre Tätigkeit entstanden sind, unter dem Betrag der Aufwandsentschädigungspauschale liegen. Im Freiwilligensurvey 2014 wird zum ersten Mal 
eine klare Trennung zwischen Kostenerstattung und Zugewinn (durch Geldzahlungen oder Sachzuwendungen) vorgenommen.

Kosten und Kostenerstattungen sowie Geldzahlungen und Sachzuwendungen werden im Freiwilligensurvey 2014 für die von den Engagierten genannte freiwillige Tätigkeit erfasst (bei Engagierten, die lediglich eine Tätigkeit ausüben), beziehungsweise für die zeitintensivste Tätigkeit der Engagierten erfasst (bei Engagierten, die zwei oder mehr freiwillige Tätigkeiten ausüben). Da alle Angaben von den Engagierten selbst berichtet werden, zum Beispiel zu den Kosten, können sich Abweichungen zu den Informationen ergeben, die etwa von zivilgesellschaftlichen Organisationen bereitgestellt werden. Beispielsweise können Kostenerstattungen möglich sein, ohne dass die Befragten diese in Anspruch nehmen oder solche in der Befragung nicht nennen, weil sie ihnen nicht bekannt sind.

Auch die Klassifikation der freiwillig Engagierten ist im Freiwilligensurvey durch die Einschätzung der Befragten bestimmt. Freiwillig engagiert ist, wer freiwillig oder ehrenamtlich Aufgaben oder Arbeiten übernommen hat, die unbezahlt oder gegen eine geringe Aufwandsentschädigung erbracht werden. Daraus resultiert, dass auch Personen, die Geld für ihr Engagement erhalten, als freiwillig engagiert betrachtet werden. Wir schließen diese auch nicht nachträglich aus den Analysen aus, weil Geldzahlungen kein hinreichender Grund hierfür sind (im Gegensatz zu den Personen, die bei der Prüfung der offenen Angaben als nicht-engagiert eingestuft werden, etwa weil sie eine berufliche Tätigkeit als Betriebsratsmitglied als freiwillige Tätigkeit angegeben haben, siehe Kapitel 2).

Kosten: In der Befragung 2014 wurde erstmalig erfasst, ob Kosten aus der freiwilligen Tätigkeit entstehen. Darüber hinaus wurde 2014 gesondert gefragt, ob eine Kostenerstattung möglich ist und gegebenenfalls ob von dieser Möglichkeit
Gebrauch gemacht wird. ${ }^{1}$ Wir gehen davon aus, dass es sich bei Inanspruchnahme dieser Kostenerstattung nicht um einen finanziellen Zugewinn handelt.

Entstehen Ihnen Kosten aus Ihrer Tätigkeit, zum Beispiel Fahrt- oder Materialkosten? Mitgliedsbeiträge und Spenden zählen Sie bitte nicht dazu.

\section{Antwortkategorien: ja; nein}

Falls ja:

Besteht prinzipiell die Möglichkeit für Sie, sich diese Kosten erstatten zu lassen?

\section{Antwortkategorien: ja; teilweise; nein}

Falls ,ja' oder ,teilweise‘ gewählt werden, folgt die Frage:

\section{Machen Sie von einer Erstattung der Kosten in der Regel Gebrauch?}

\section{Antwortkategorien: ja; nein}

In der Analyse werden die Antwortkategorien ,ja und ,teilweise zusammengefasst und beide als ,Gebrauch Kostenerstattung' ausgewertet.

1 In früheren Wellen des Freiwilligensurveys wurde den freiwillig Engagierten die folgende Frage zur Kostenerstattung gestellt: „Können Sie für finanzielle Auslagen, die Sie im Zusammenhang mit Ihrer Tätigkeit haben, gegen Nachweis eine Kostenerstattung erhalten?" (Antwortkategorien: ja; nein; trifft nicht zu). Bei dieser Frage bleibt unklar, ob den Engagierten überhaupt Kosten entstanden waren oder nicht. Es gab zwei Folgefragen, eine nach der Inanspruchnahme der Kostenerstattung („Machen Sie davon regelmäßig oder gelegentlich Gebrauch?", Antwortkategorien: ja, regelmäßig; ja, gelegentlich; nein) und eine Frage nach Erhalt einer pauschalierten Aufwandsentschädigung (siehe Tabelle 14.1). Dabei blieb allerdings unklar, ob die Personen zwischen Kostenerstattung und pauschalierter Aufwandsentschädigung unterschieden haben. 
Geldzahlungen und Sachzuwendungen: Um einen finanziellen Zugewinn gesondert zu erfassen, wurden in der Erhebung 2014 der Erhalt von Geldzahlungen und der erhaltene Betrag sowie der Erhalt von Sachzuwendungen folgendermaßen erfragt, und zwar, nachdem die Fragen zu Kostenentstehung und -erstattung gestellt waren:

Unabhängig von einer möglichen Kostenerstattung: Haben Sie persönlich in den vergangenen 12 Monaten Geld für Ihre freiwillige Tätigkeit erhalten?

\section{Antwortkategorien: ja; nein}

Wenn ja, folgte die Frage zur Höhe des Betrages, beginnend mit:

Wenn Sie an die letzten 12 Monate denken: Wie viel Geld haben Sie im Durchschnitt pro Monat erhalten: bis einschließlich 150 Euro oder über 150 Euro?

Je nachdem wie die Antwort lautete, wurden weitere Beträge abgefragt (Tabelle 14-1). In der Analyse wurden die Geldbeträge in drei Gruppen zusammengefasst, weil nur wenige Personen über 350 Euro beziehungsweise über 500 Euro erhalten: ,bis 50 Euro, ,51 bis 150 Euro und ,mehr als 150 Euró.

Der Erhalt von Sachzuwendungen wurde folgendermaßen erhoben:

Wenn Sie an die letzten 12 Monate denken: Haben Sie im Rahmen Ihrer Tätigkeit Sachzuwendungen erhalten, zum Beispiel Fahrscheine oder eine kostenlose private Nutzung von Räumen oder Ausstattungsmitteln?

\section{Antwortkategorien: ja; nein}

Die Erhebung der Vergütung wurde von Welle zu Welle geändert. Daher sind die Anteile in den vier Erhebungsjahren nicht uneingeschränkt vergleichbar. In den Jahren 1999 und 2009 wurde zwischen, einer pauschalierten Aufwandsentschädigung', ,Honoraren', ,einer geringfügigen Bezahlung' und ,Sachzuwendungen' differenziert. Im Jahr 2004 wurden die Information zur Höhe der Geldzahlungen sowie die Sachzuwendungen nicht erfasst (Tabelle 14-1).

Die drei Kategorien , pauschalierte Aufwandsentschädigungen, ,Honorare ' und ,geringfügige Bezahlungen' aus den Jahren 1999, 2004 und 2009 sind in der nachfolgenden Analyse addiert worden, um den Vergleich mit 2014 zu ermöglichen (Tabelle 14-2). Allerdings wird durch diese Vorgehensweise die Bedeutung der Geldzahlungen im freiwilligen Engagement in den Jahren 1999, 2004 und 2009 möglicherweise überschätzt, denn pauschalierte Aufwandsentschädigungen müssen keinen finanziellen Zugewinn bedeuten, sie können reine Kostenerstattungen darstellen. Aufgrund der konzeptuellen Unklarheit sind pauschalierte Aufwandsentschädigungen in Tabelle 14-2 sowohl in der Kategorie der Kostenerstattung als auch in der Kategorie des finanziellen Zugewinns aufgeführt. Vorteil der präzisierten Abfrage 2014 ist, dass ausschließlich Geldzahlungen, die als finanzieller Zugewinn gewertet werden können, als solche erhoben werden, und nicht Aufwandsentschädigungen mit eingerechnet werden, die zwar meist nur die Kosten abdecken sollen, in manchen Fällen aber auch einen finanziellen Zugewinn für die Engagierten darstellen können (wenn die Pauschale höher liegt als der Aufwand).

In den Befragungen von 1999, 2004 und 2009 sind die pauschalierten Aufwandsentschädigungen jeweils die am häufigsten genannte Kategorie mit 5,5 Prozent im Jahr 1999, 7,4 Prozent im Jahr 2004 und 9,7 Prozent im Jahr 2009, die geringfügige Bezahlung steht jeweils an zweiter Position und am seltensten sind jeweils Honorare. 
Tabelle 14-1: Erfassung der Geldzahlungen im Zeitvergleich

\begin{tabular}{|c|c|c|c|}
\hline Welle & Fragetext & $\begin{array}{l}\text { Antwort- } \\
\text { möglich- } \\
\text { keiten }\end{array}$ & $\begin{array}{l}\text { Wenn die Antwort, ja‘ lautete, } \\
\text { wurden folgende Beträge } \\
\text { abgefragt* }\end{array}$ \\
\hline 1999 & $\begin{array}{l}\text { Erhalten Sie persönlich eine gewisse Vergütung, } \\
\text { beispielsweise eine pauschalierte Aufwandsent- } \\
\text { schädigung (1), Honorare (2), Eine geringfügige } \\
\text { Bezahlung (3), Sachzuwendungen, z. B. Fahrschei- } \\
\text { ne, private Nutzung von Gruppenräumen oder } \\
\text { Ausstattungsmitteln (4), Nein, nichts davon (5). } \\
\text { (Mehrfachnennungen sind möglich) }\end{array}$ & $\mathrm{Ja} /$ Nein & $\begin{array}{l}\text { Unter } 300 \text { DM/oder über } 300 \text { DM: } \\
\text { Und je nach Antwort: } \\
\text { Unter } 100 \text { DM/oder über } 100 \text { DM } \\
\text { Oder: } \\
\text { Unter } 700 \text { DM/oder über } 700 \text { DM }\end{array}$ \\
\hline 2004 & $\begin{array}{l}\text { Erhalten Sie persönlich eine gewisse Vergütung, } \\
\text { beispielsweise eine pauschalierte Aufwandsent- } \\
\text { schädigung (1), Honorare (2), eine geringfügige } \\
\text { Bezahlung (3), Nein, nichts trifft davon zu (4). } \\
\text { (Mehrfachnennungen sind möglich) }\end{array}$ & Ja/Nein & $\begin{array}{l}\text { Keine Abfrage } \\
\text { der Beträge }\end{array}$ \\
\hline 2009 & $\begin{array}{l}\text { Erhalten Sie persönlich eine gewisse Vergütung, } \\
\text { beispielsweise eine pauschalierte Aufwandsent- } \\
\text { schädigung (1), Honorare (2), eine geringfügige } \\
\text { Bezahlung (3), Sachzuwendungen (4), Nein, nichts } \\
\text { davon (5), Keine Angabe (6). } \\
\text { (Mehrfachnennungen sind möglich) }\end{array}$ & Ja/Nein & $\begin{array}{l}\text { Unter } 150 \text { Euro/oder über } 150 \text { Euro } \\
\text { Und je nach Antwort: } \\
\text { Unter } 50 \text { Euro/oder über } 50 \text { Euro } \\
\text { Oder: } \\
\text { Unter } 350 \text { Euro/oder über } 350 \text { Euro } \\
\text { Und: } \\
\text { Unter } 500 \text { Euro/oder über } 500 \text { Euro }\end{array}$ \\
\hline 2014 & $\begin{array}{l}\text { Unabhängig von einer möglichen Kostenerstat- } \\
\text { tung: Haben Sie persönlich in den vergangenen } \\
12 \text { Monaten Geld für Ihre freiwillige Tätigkeit } \\
\text { erhalten? } \\
\text { Sachzuwendungen wurden separat erfragt (siehe } \\
\text { oben) }\end{array}$ & Ja/Nein & $\begin{array}{l}150 \text { Euro/über } 150 \text { Euro } \\
\text { Und je nach Antwort: } \\
\text { Bis einschl. } 50 \text { Euro/über } 50 \text { Euro } \\
\text { Oder: } \\
\text { Bis einschl. } 350 \text { Euro/über } 350 \text { Euro } \\
\text { Und: } \\
\text { Bis einschl. } 500 \text { Euro/über } 500 \text { Euro }\end{array}$ \\
\hline
\end{tabular}

Quelle: FWS 1999, FWS 2004, FWS 2009, FWS 2014. Eigene Darstellung (DZA). Anmerkungen: * Die Abfrage der Beträge wurde gefiltert, dass heißt, nicht allen Befragten wurden alle Beträge genannt, sondern nur die Kategorie(n), die der zuvor gegebenen Antwort entsprechen.

Tabelle 14-2: Geldzahlungen und Sachzuwendungen für die freiwillige Tätigkeit im Zeitvergleich

\begin{tabular}{|c|c|c|c|c|}
\hline & 1999 & 2004 & 2009 & 2014 \\
\hline $\begin{array}{l}\text { Erstattung } \\
\text { entstandener } \\
\text { Kosten } \\
\end{array}$ & \multicolumn{3}{|c|}{$\begin{array}{l}\text { Kostenerstattung gegen Nachweis } \\
\text { (bzw. pauschalierte Aufwandsentschädigung) }\end{array}$} & $\begin{array}{l}\text { Kostenerstattungs- } \\
\text { möglichkeit und } \\
\text {-inanspruchnahme }\end{array}$ \\
\hline $\begin{array}{l}\text { Zugewinn: } \\
\text { Geldzahlung }\end{array}$ & \multicolumn{3}{|c|}{$\begin{array}{l}\text { (Pauschalierte Aufwandsentschädigungen), } \\
\text { Honorare und geringfügige Bezahlungen }\end{array}$} & Geldzahlungen \\
\hline $\begin{array}{l}\text { Zugewinn: } \\
\text { Sachleistung }\end{array}$ & Sachzuwendungen & Nicht erfasst & Sachzuwendungen & Sachzuwendungen \\
\hline
\end{tabular}

Quelle: FWS 1999, FWS 2004, FWS 2009, FWS 2014. Eigene Darstellung (DZA). 


\subsection{Geldzahlungen und Sachzuwendungen}

\subsubsection{Geldzahlungen und Sachzuwendungen 2014}

Zunächst betrachten wir den Anteil der Engagierten, die 2014 angeben, dass sie Geldzahlungen für ihre Tätigkeit erhalten haben (Abbildung 14-1). Der größte Anteil der engagierten Frauen und Männer erhält keine Geldzahlung. Lediglich knapp jede beziehungsweise jeder Zehnte erhält Geld im freiwilligen Engagement. Männer (11,1 Prozent) erhalten hierbei etwas häufiger Zahlungen als Frauen (8,7 Prozent). Engagierte im Alter von 14 bis 29 Jahren erhalten mit einem Anteil von 13,7 Prozent am häufigsten Geld für ihre freiwillige Tätigkeit (Abbildung 14-1a). In den Altersgruppen der 30- bis 49-jährigen Engagierten und der 50- bis 64-jährigen Engagierten liegen die entsprechenden Anteile mit 9,5 Prozent und 8,6 Prozent ebenso darunter wie bei den 65 Jahre alten und älteren Engagierten mit 8,0 Prozent. Zudem zeigen sich Unterschiede zwischen den Bildungsgruppen (Abbildung 14-1b), am häufigsten erhalten Schülerinnen und Schüler Geld für ihre freiwillige Tätigkeit (10,8 Prozent) und am seltensten Personen mit niedriger Bildung (8,1 Prozent). 
Abbildung 14-1: Anteile der Engagierten, die Geldzahlungen für die freiwillige Tätigkeit erhalten, 2014, a) gesamt, nach Geschlecht und nach Alter und b) nach Bildung

a) gesamt, nach Geschlecht und nach Alter

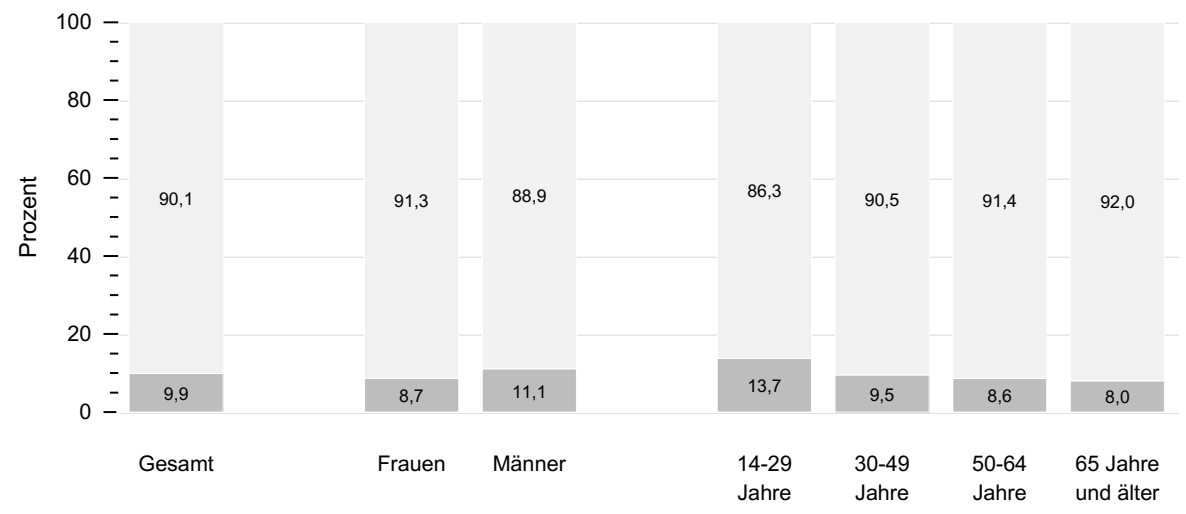

b) nach Bildung

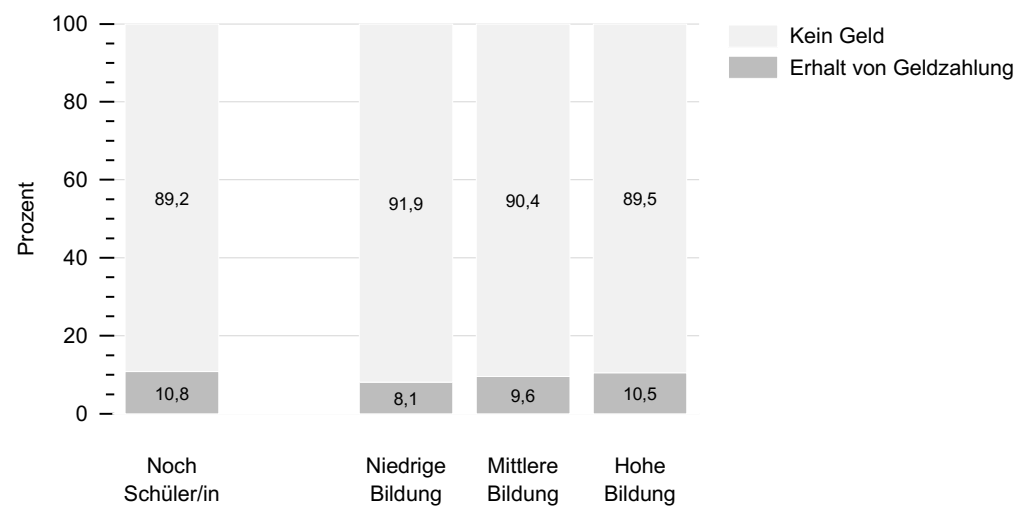

Quelle: FWS 2014, gewichtet, eigene Berechnungen (DZA). Basis: Alle Engagierten. Gesamt, Alter und Geschlecht $(n=12.417)$, Bildung $(n=12.414)$. 
Die Engagierten, die Geldzahlungen erhalten, bekommen in der Regel niedrige Beträge. Von allen Engagierten erhalten 5,1 Prozent eine Zahlung von maximal bis zu 50 Euro im Monat (Abbildung 14-2a). Lediglich 1,3 Prozent erhalten einen Betrag, der einer Summe von über 150 Euro monatlich entspricht. Nur 1,0 Prozent der engagierten Frauen und 1,7 Prozent der engagierten Männer erhalten Geldzahlungen von über 150 Euro pro Monat. Die 14- bis 29-Jährigen erhalten am häufigsten kleine Beträge von maximal bis zu 50 Euro pro Monat. Kaum Differenzen sind bei dem Anteil der Engagierten, die große Geldzahlungen bekommen, zwischen den Bildungsgruppen zu beobachten (Abbildung 14-2b).
Im Freiwilligensurvey wird nicht nur nach Geldzahlungen sondern auch nach Sachzuwendungen (wie zum Beispiel die kostenlose private Nutzung von Räumen oder Ausstattungsmitteln) gefragt. Diese Sachzuwendungen können parallel zu Geldzahlungen als eine geldwerte Vergütung des Engagements betrachtet werden. In der Vergangenheit wurden Sachzuwendungen als eine "eher unproblematische Form der Vergütung" eingestuft, weil sie Engagement einerseits motivieren, befördern und ermöglichen können, andererseits aber keine Konkurrenz zwischen Engagement und Erwerbsarbeit beziehungsweise Gewinnorientierung darstellen (Gensicke 2015: 257). 
Abbildung 14-2: Anteile der Engagierten, die Geldzahlungen für die freiwillige Tätigkeit erhalten, differenziert nach der Höhe der monatlichen Beträge, 2014, a) gesamt, nach Geschlecht und nach Alter und b) nach Bildung

a) gesamt, nach Geschlecht und nach Alter

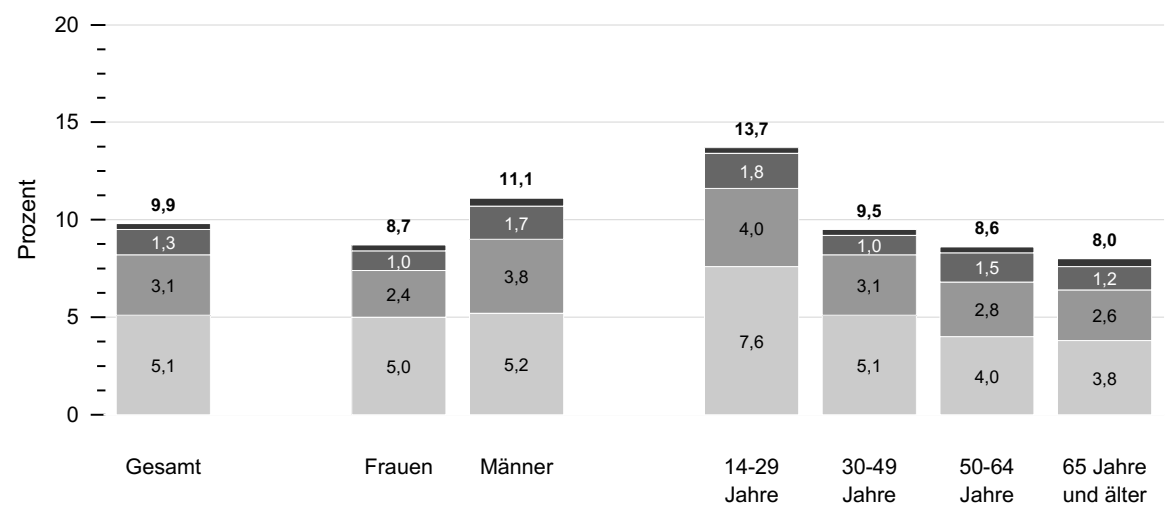

b) nach Bildung

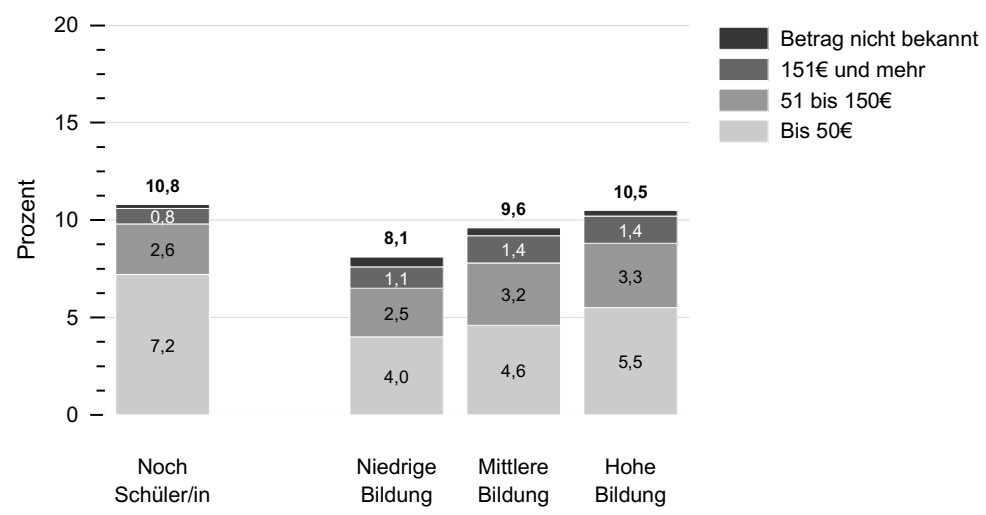

Quelle: FWS 2014, gewichtet, eigene Berechnungen (DZA). Basis: Alle Engagierten. Gesamt, Alter und Geschlecht $(\mathrm{n}=12.417)$, Bildung $(\mathrm{n}=12.414)$. 
Der Anteil der Engagierten, die im Jahr 2014 Sachzuwendungen erhalten, ist mit 14,6 Prozent gering (Abbildung 14-3). Das ist nur geringfügig mehr als der Anteil der Engagierten, die Geld erhalten. Der Anteil von Personen, die Sachzuwendungen erhalten, unterscheidet sich nicht signifikant zwischen Frauen und Männern. Während Sachzuwendungen im Alter ab
65 Jahren mit einem Anteil von 7,3 Prozent lediglich eine geringfügige Rolle spielen und auch bei den 30 - bis 49-Jährigen sowie den 50 - bis 64-Jährigen unterdurchschnittlich anfallen, sind sie insbesondere bei den jungen Menschen von großer Bedeutung: 32,1 Prozent der Engagierten im Alter von 14 bis 29 Jahren erhalten solche Zuwendungen.

Abbildung 14-3: Anteile der Engagierten, die Sachzuwendungen für die freiwillige Tätigkeit erhalten, 2014, a) gesamt, nach Geschlecht und nach Alter und b) nach Bildung

a) gesamt, nach Geschlecht und nach Alter

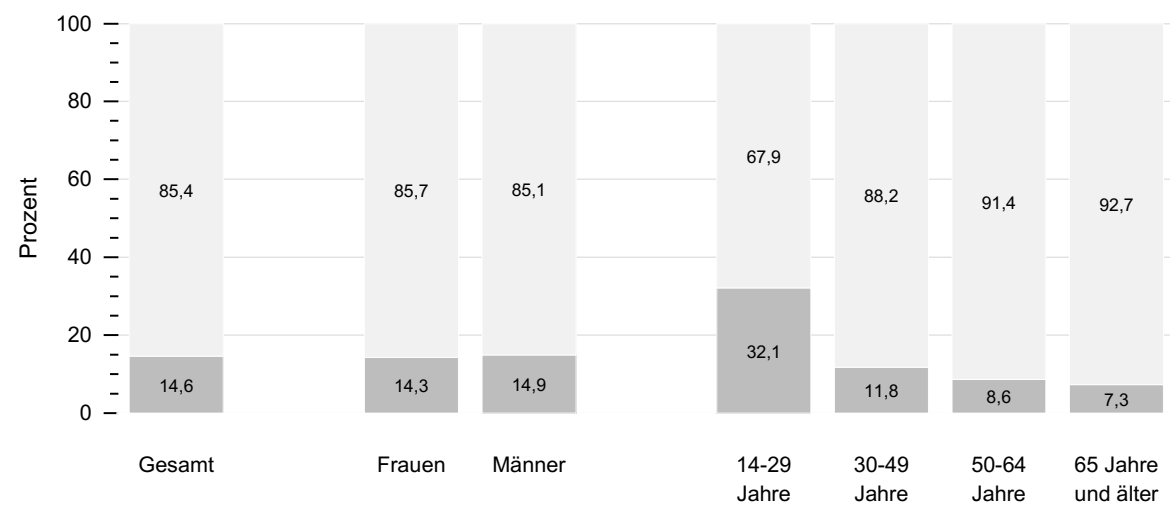

\section{b) nach Bildung}

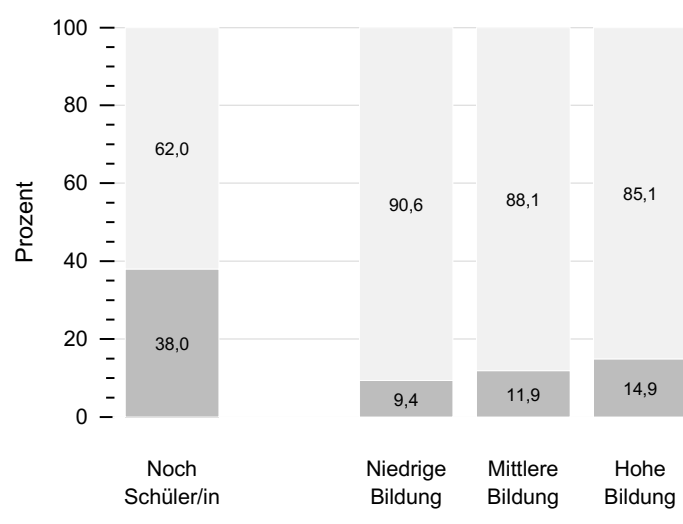

Quelle: FWS 2014, gewichtet, eigene Berechnungen (DZA). Basis: Alle Engagierten. Gesamt, Alter und Geschlecht $(n=12.415)$, Bildung $(n=12.412)$. 
Bezüglich dieses Phänomens sind dementsprechend auch die Unterschiede zwischen den Bildungsgruppen größer als bei den Geldzahlungen. Der Anteil der freiwillig engagierten Schülerinnen und Schülern, die Sachzuwendungen erhalten, ist mit 38,0 Prozent überdurchschnittlich hoch (Abbildung 14-3b). Während 15,3 Prozent der engagierten Frauen und 14,5 Prozent der engagierten Männer mit einem hohen Bildungsabschluss Sachzuwendungen erhalten, ist das nur für 8,9 Prozent der Frauen und
9,7 Prozent der Männer mit einem niedrigen Bildungsabschluss der Fall (Abbildung 14-4). Sachzuwendungen scheinen somit insbesondere für die Gruppe der Schülerinnen (35,9 Prozent) und für die Gruppe der Schüler (40,2 Prozent) bei der Ausübung einer freiwilligen Tätigkeit ins Gewicht zu fallen (Abbildung 14-4). Unter den Schülerinnen und Schüler sind es insbesondere diejenigen, die auf dem Gymnasium sind, die am häufigsten Sachzuwendungen erhalten (ohne Abbildung).

Abbildung 14-4: Anteile der Engagierten, die Sachzuwendungen für die freiwillige Tätigkeit erhalten, 2014, gesamt, nach Bildung und Geschlecht

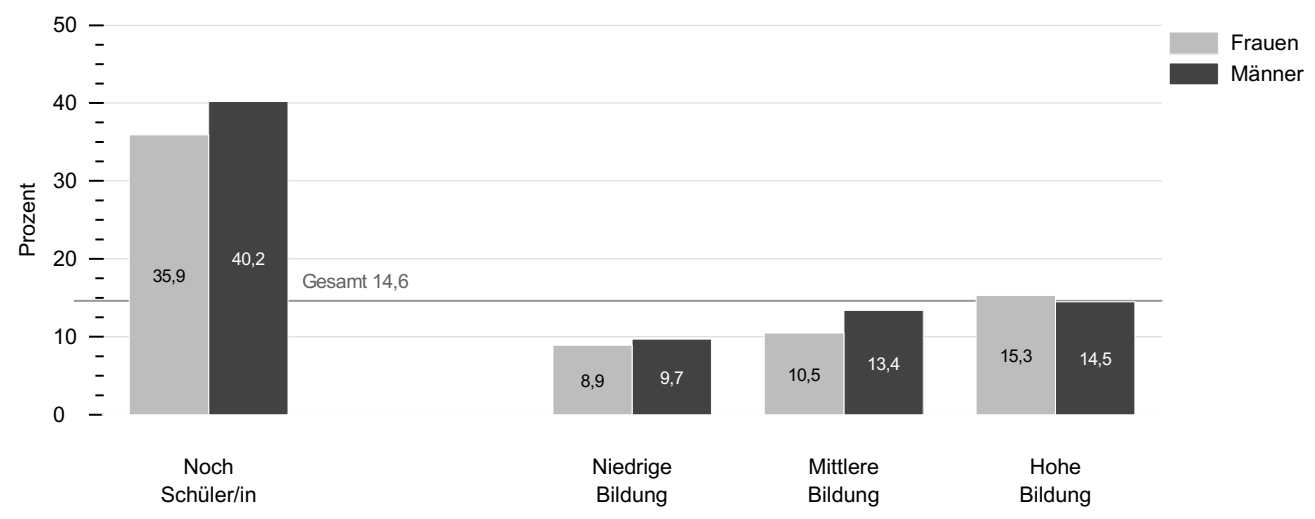

Quelle: FWS 2014, gewichtet, eigene Berechnungen (DZA). Basis: Alle Engagierten $(n=12.412$ ). 


\subsubsection{Geldzahlungen und Sach- zuwendungen in den gesellschaftlichen Bereichen des Engagements}

Die Wahrscheinlichkeit, Geldzahlungen für freiwillige Tätigkeiten zu erhalten, variiert mit dem gesellschaftlichen Bereich, in dem sich Engagierte betätigen. Am häufigsten (44,1 Prozent) erhalten Engagierte Geldzahlungen, die im Bereich Justiz und Kriminalitätsprobleme freiwillig tätig sind (Abbildung 14-5). Auch in den Bereichen Politik (24,8 Prozent), Unfall- und Rettungsdienst oder Freiwillige Feuerwehr (20,7 Pro- zent) und Jugendarbeit oder Bildungsarbeit für Erwachsene (17,1 Prozent) sind Geldzahlungen weiter verbreitet als in den anderen Bereichen. Zudem erhalten immerhin 16,0 Prozent der Engagierten im Gesundheitsbereich Geld für ihre Tätigkeit. Schule und Kindergarten sowie Kirche und Religion sind dagegen Bereiche, in denen es selten Geld für Engagierte gibt. Einerseits ist dies offensichtlich auch entlang von Organisationstraditionen historisch so gewachsen (etwa im Bereich Kirche und Religion), andererseits fehlen zum Beispiel bei den öffentlichen Trägern von Kindergärten und Schulen häufig die finanziellen Mittel, um Freiwillige bezahlen zu können (Klie 2015).

Abbildung 14-5: Anteile der Engagierten, die Geldzahlungen für die freiwillige Tätigkeit erhalten, 2014, nach Bereichen

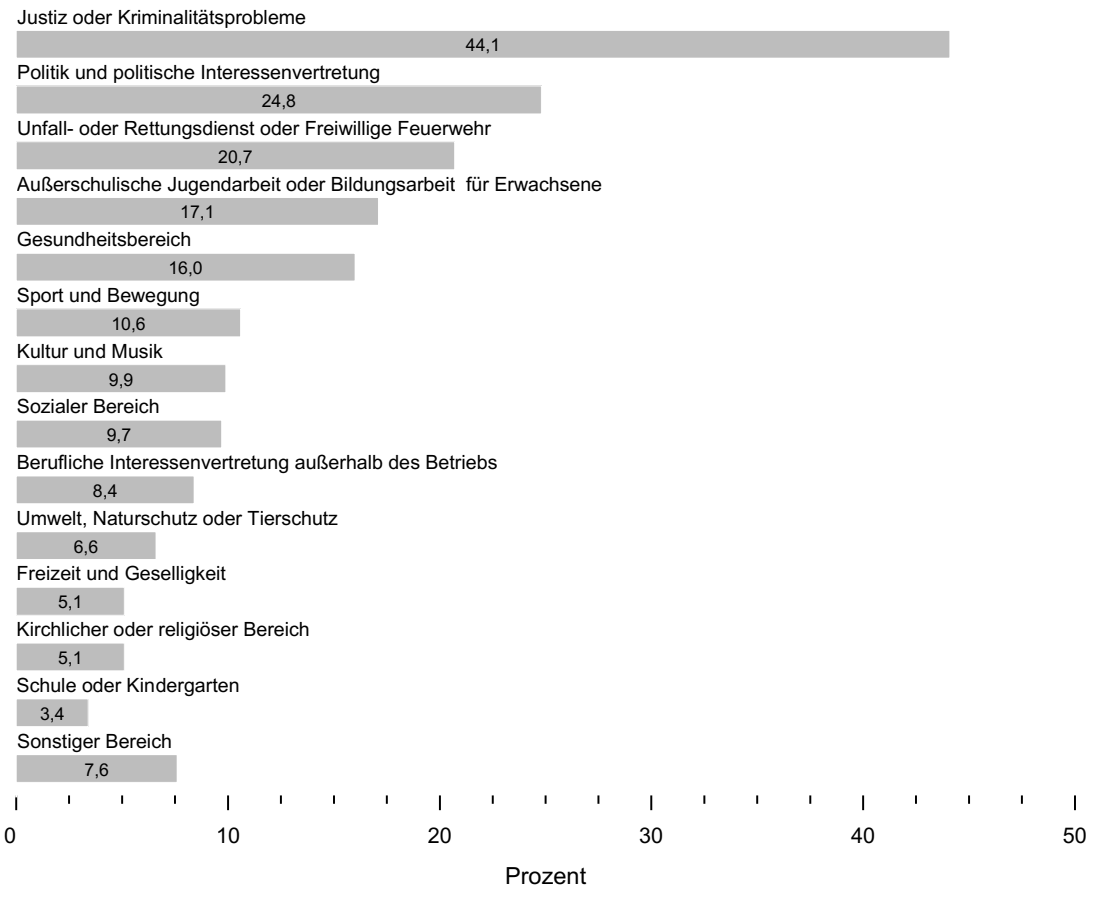

Quelle: FWS 2014, gewichtet, eigene Berechnungen (DZA). Basis: Alle Engagierten ( $n=12.328)$. 
In Abbildung 14-6 sehen wir auch, dass Geschlechterunterschiede bei den Geldzahlungen zwischen den Bereichen existieren. In fast allen Bereichen ist der Anteil von Männern, die Geldzahlungen für ihr Engagement erhalten, höher als der Anteil von Frauen. Im Gesundheitsbereich und bei den Unfall- und Rettungsdiensten sind die Differenzen zwischen Männern und Frauen am höchsten (6,3 und 5,1 Prozentpunkte). Als eine der wenigen Ausnahmen erhalten im Bereich Sport anteilig mehr Frauen als Männer Geldzahlungen (Unterschied von 3,3 Prozentpunkten).

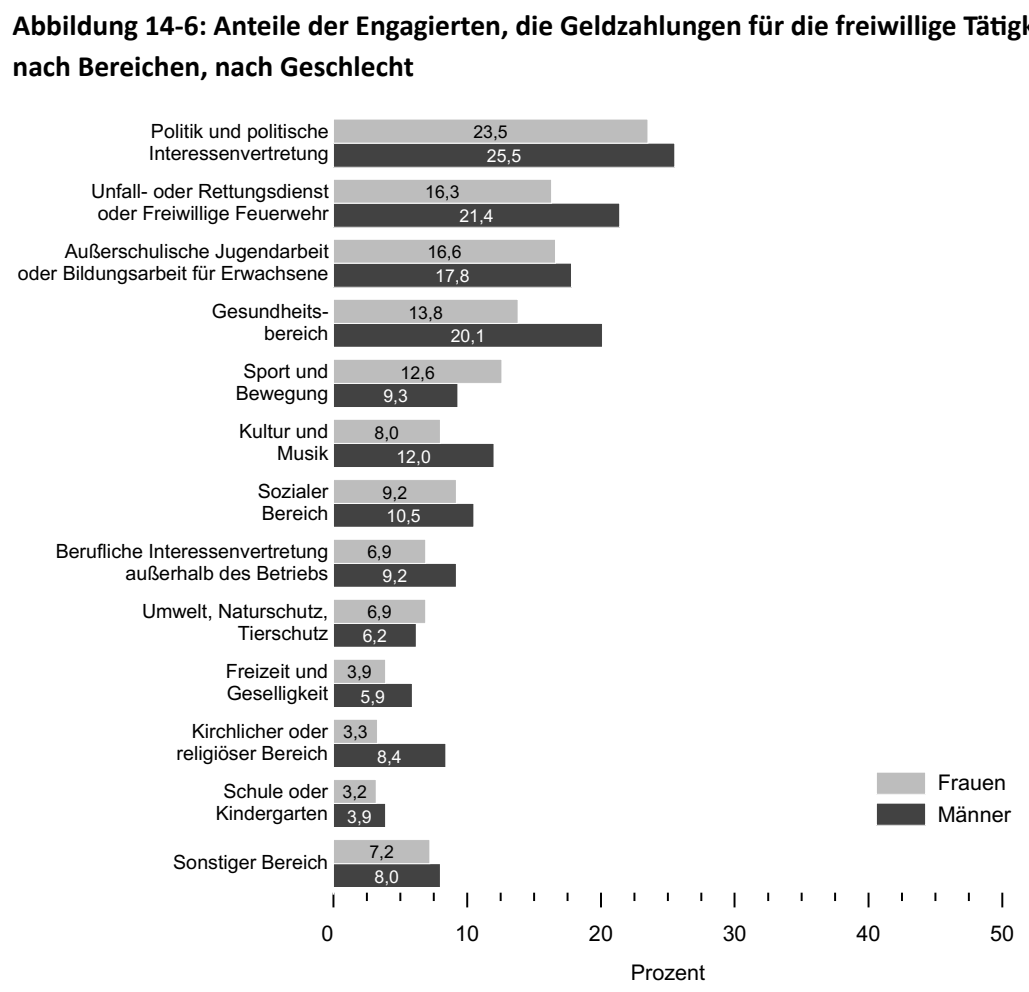

Quelle: FWS 2014, gewichtet, eigene Berechnungen (DZA). Basis: Alle Engagierten $(n=12.254)$.

Für den Bereich ,Justiz oder Kriminalitätsprobleme' werden keine Ergebnisbalken nach Geschlecht dargestellt, da im Datensatz jeweils weniger als 50 Fälle engagierter Frauen und Männer in diesem Bereich enthalten sind. 
Bei den Sachzuwendungen zeigen sich andere Verteilungen (Abbildung 14-7). Im Bereich Jugendarbeit werden häufiger Sachzuwendungen als in allen anderen Bereichen bereitgestellt (22,7 Prozent). In diesem Bereich werden zum Beispiel Aufenthalts- und Übungsräume häufig von den Engagierten benötigt, ebenso wie im Bereich Kultur und Musik (19,1 Prozent), in dem zum Beispiel auch Instrumente zur Verfügung gestellt werden, die auch privat genutzt werden können.
Im Gesundheitsbereich (18,4 Prozent) werden ebenfalls überdurchschnittlich häufig Sachzuwendungen an freiwillig Engagierte geleistet. Zum Beispiel sind in diesem Bereich Gutscheine für Trainingsstunden gängig, die von den Engagierten auch privat genutzt werden können. Im Bereich Unfall- und Rettungsdienst (17,2 Prozent) muss auch die notwendige Infrastruktur wie zum Beispiel durch Fahrzeuge und Kommunikationsmittel bereitgestellt werden.

\section{Abbildung 14-7: Anteile der Engagierten, die Sachzuwendungen für die freiwillige Tätigkeit erhalten, 2014, nach Bereichen}

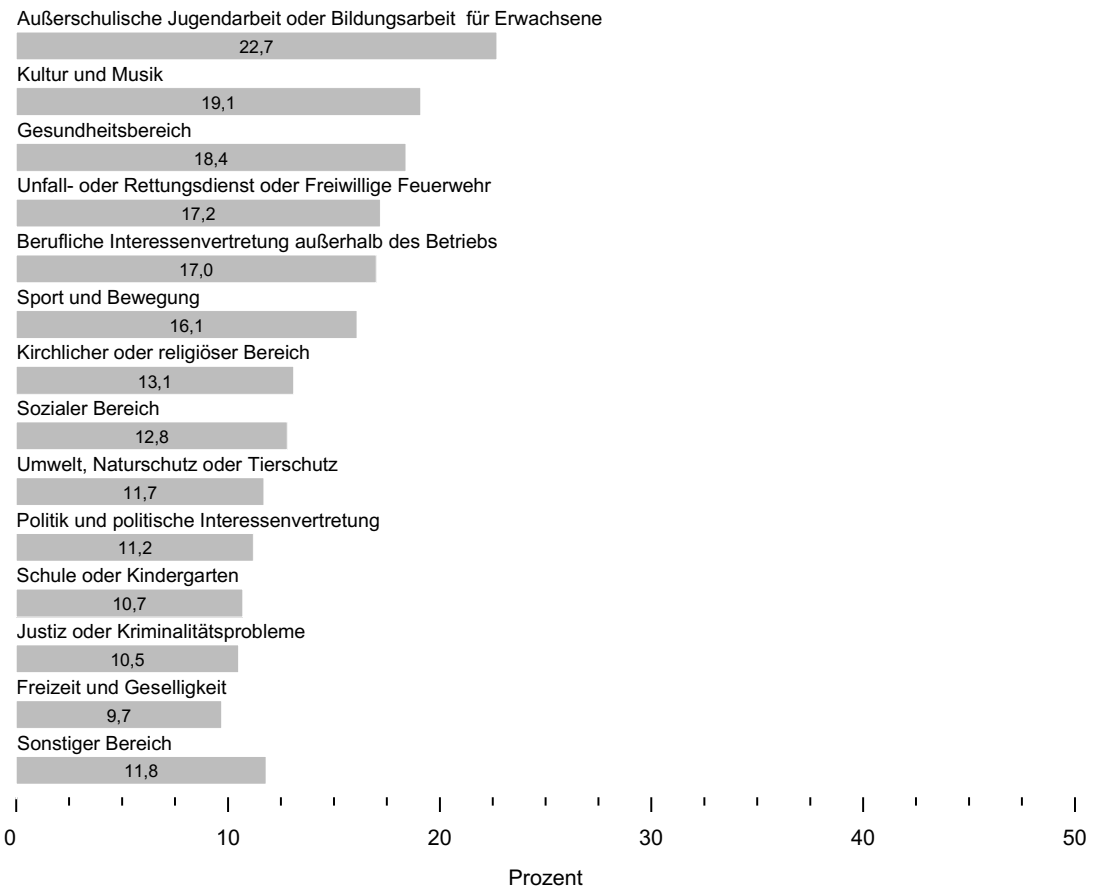

Quelle: FWS 2014, gewichtet, eigene Berechnungen (DZA). Basis: Alle Engagierten $(n=12.325)$. 
In den meisten Bereichen erhalten Frauen häufiger Sachzuwendungen als Männer (Abbildung 14-8). Das ist in der Jugendarbeit, bei Unfalloder Rettungsdiensten, der beruflichen Interessenvertretung, im Sport, im Umwelt, Natur- oder Tierschutz und in der Politik und der politischen Interessenvertretung der Fall. Der höchste Un- terschied von 11,0 Prozentpunkten ist in dem Bereich berufliche Interessenvertretung $\mathrm{zu}$ beobachten, hier erhalten mehr Frauen als Männer Sachzuwendungen. Im Gesundheitsbereich zeigt sich ein anderes Bild, in diesem erhalten Männer mehr Sachzuwendungen als Frauen (8,6 Prozentpunkte Unterschied).

\section{Abbildung 14-8: Anteile der Engagierten, die Sachzuwendungen für die freiwillige Tätigkeit erhalten, 2014, nach Bereichen, nach Geschlecht}

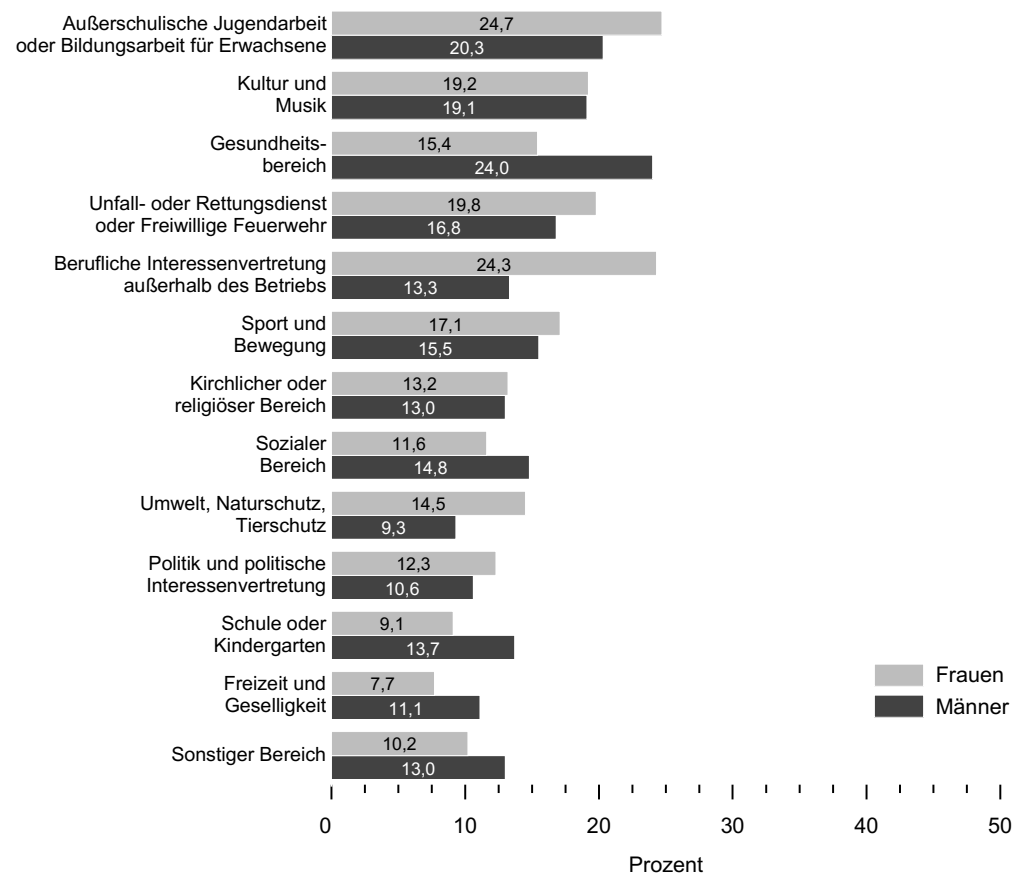

Quelle: FWS 2014, gewichtet, eigene Berechnungen (DZA). Basis: Alle Engagierten $(n=12.251)$.

Für den Bereich ,Justiz oder Kriminalitätsprobleme' werden keine Ergebnisbalken nach Geschlecht dargestellt, da im Datensatz jeweils weniger als 50 Fälle engagierter Frauen und Männer in diesem Bereich enthalten sind. 
Diese Geschlechterunterschiede bei den Geldzahlungen und Sachzuwendungen korrespondieren mit den unterschiedlichen Funktionen von Männern und Frauen in ihrem freiwilligen Engagement. Frauen und Männer in Leitungsund Vorstandsfunktionen erhalten dabei ähnlich häufig Geldzahlungen und/oder Sachzuwendungen (26,6 Prozent und 25,1 Prozent, Abbildung 14-9). Eine größere Differenz ist bei den Frauen und Männern ohne Leitungs- und Vorstands- funktion $\mathrm{zu}$ beobachten. Mehr Männer ohne eine solche Funktion erhalten Geldzahlungen und/oder Sachzuwendungen (22,6 Prozent) als Frauen ohne eine solche Funktion (19,4 Prozent). Darüber hinaus gibt es häufiger Männer in Leitungspositionen (siehe Kapitel 11) als Frauen, sodass der Gesamtanteil von Frauen, die Geldzahlungen und/oder Sachzuwendungen erhalten, niedriger ist.

\section{Abbildung 14-9: Anteile der Engagierten, die Geldzahlungen und/oder Sachzuwendungen für die freiwillige} Tätigkeit erhalten, 2014, nach Leitungsfunktion

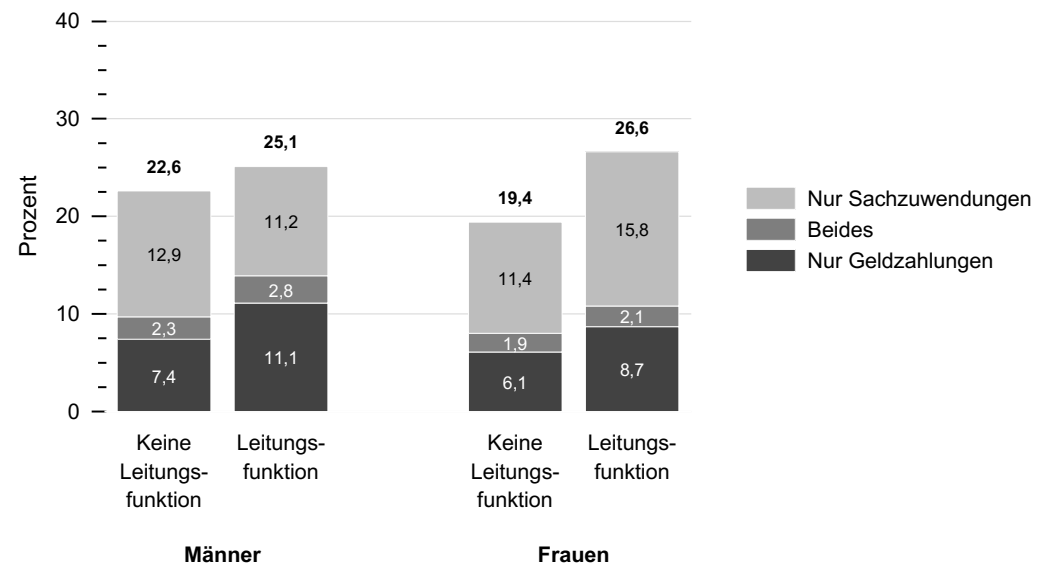

Quelle: FWS 2014, gewichtet, eigene Berechnungen (DZA). Basis: Alle Engagierten ( $n=12.393$ ). 
Anhand der Befunde in Abbildung 14-10 lässt sich zudem zeigen, in welchen Bereichen für die Gruppe der Schülerinnen und Schüler besonders häufig Sachzuwendungen bereitgestellt werden. Dies ist der Fall im Bereich Kultur und Musik, 51,5 Prozent der Schülerinnen und Schüler, die sich in diesem Bereich engagieren, erhalten Sachzuwendungen, zu denen außer Übungsräumen für Theater beispielsweise auch Übungsinstrumente oder Freitickets für Kulturveranstaltungen zählen, wobei die private Nutzung sich sicherlich nicht immer eindeutig abgrenzen lässt von der
Nutzung im Rahmen der freiwilligen Tätigkeit. Zudem ist im Bereich Schule und Kindergarten (44,6 Prozent) der Anteil von Schülerinnen und Schülern, die Sachzuwendungen erhalten, überdurchschnittlich hoch. Hier ist auch an Materialien für Schulprojekte zu denken wie Farben oder Bastelmaterial. Viele dieser Sachzuwendungen sind sehr wahrscheinlich beides, eine Art Vergütung für Engagement, weil sie kostenlos genutzt werden dürfen, aber auch eine Förderung von Engagement.

Abbildung 14-10: Anteile der Engagierten, die Sachzuwendungen für die freiwillige Tätigkeit erhalten, 2014, nach ausgewählten Bereichen, nach Bildung
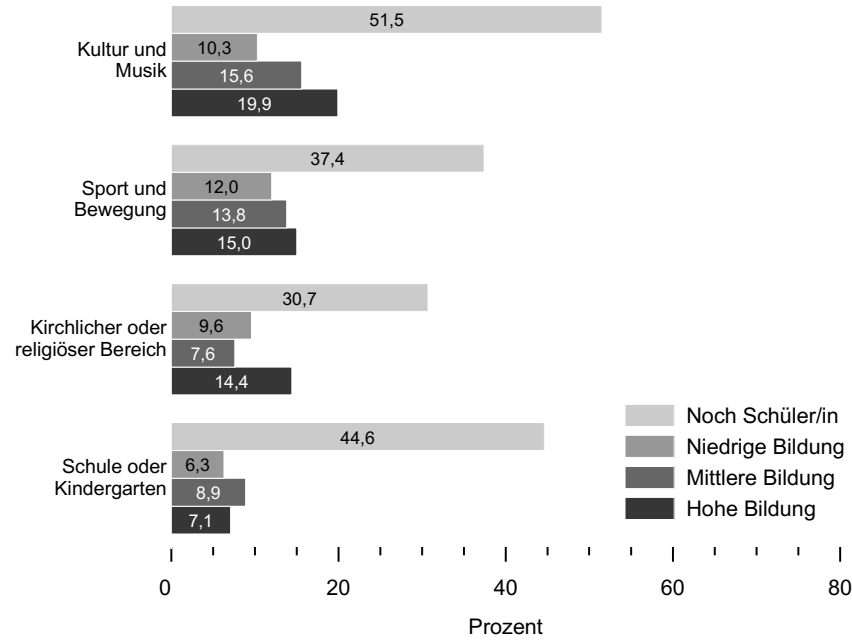

Quelle: FWS 2014, gewichtet, eigene Berechnungen (DZA). Basis: Alle Engagierten in den ausgewählten Bereichen $(n=6.918)$. 
Geld- und Sachleistungen werden meist nicht kombiniert, sondern in der Regel separat bereitgestellt, das heißt, die freiwillig Engagierten erhalten in der Regel entweder nur Geld oder nur Sachzuwendungen (Abbildung 14-11). Selbst bei den Schülerinnen und Schülern, bei denen 38,0 Prozent Sachzuwendungen erhalten sowie 10,8 Prozent Geldleistungen, ist die Kombination der beiden Leistungen selten: Lediglich rund fünf Prozent der engagierten Schülerinnen und Schüler erhalten Geld- und Sachzuwendungen für dieselbe freiwillige Tätigkeit (ohne Abbildung). Offensichtlich hängen diese Vergütungsformen von unterschiedlichen finanziellen Möglichkeiten der jeweiligen Einrichtungen und Organisationen ab, aber auch von den unterschiedlichen Tätigkeitsbereichen mit unterschiedlichen Gewohnheiten. Durch individuelle Merkmale der Engagierten allein lassen sich unterschiedliche Vergütungsformen im Engagement sicherlich nicht erklären.

Abbildung 14-11: Anteile der Engagierten, die Geldzahlungen und/oder Sachzuwendungen für die freiwillige Tätigkeit erhalten, 2014, nach Bereichen

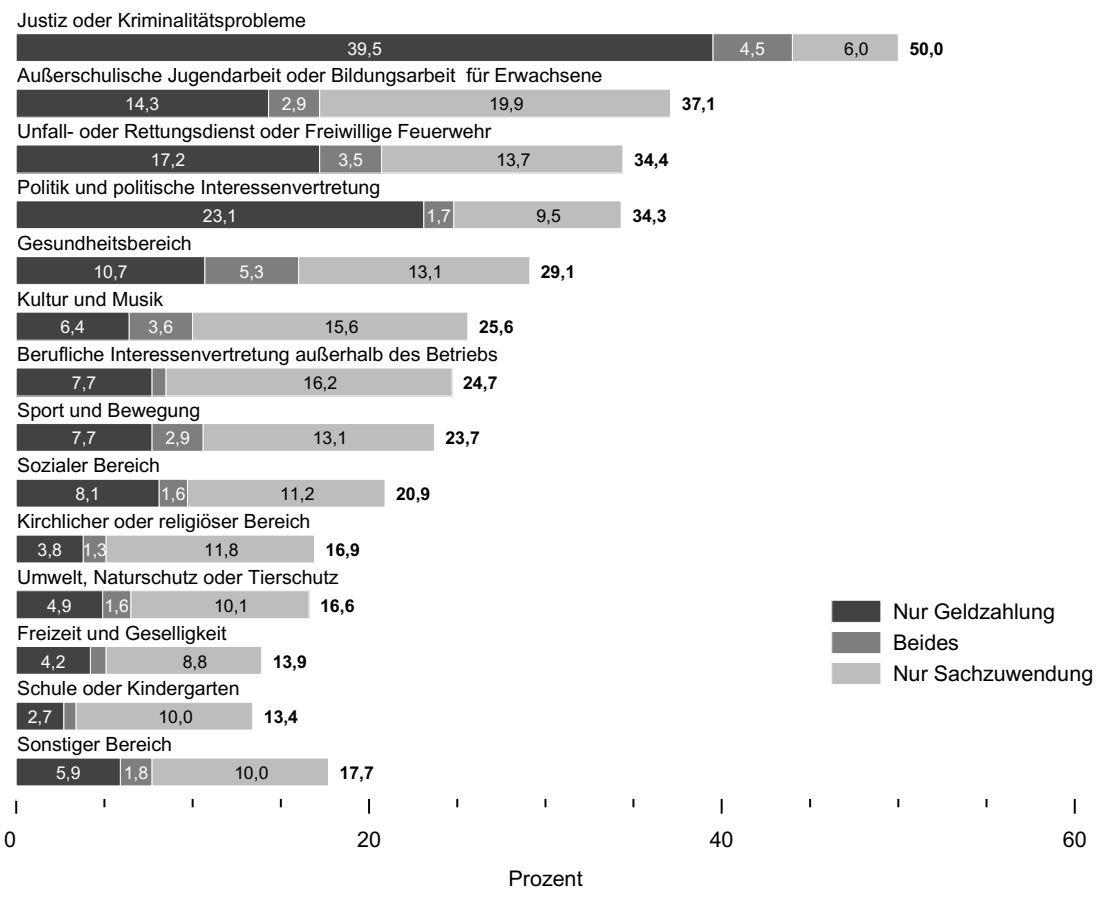

Quelle: FWS 2014, gewichtet, eigene Berechnungen (DZA). Basis: Alle Engagierten ( $n=12.319$ ). 


\subsubsection{Geldzahlungen und Sachzuwendungen im Zeitvergleich}

Wir stellen fest, dass der Anteil an Engagierten, die Geldzahlungen für das freiwillige Engagement erhalten, im Jahr 2014 gering ist (9,9 Prozent). Im Vergleich zu 1999 hat dieser Anteil sogar abgenommen (Abbildung 14-12). Zu diesem Zeitpunkt erhielten 13,4 Prozent der Engagierten Geldzahlungen. Zu berücksichtigen ist hierbei, dass die Werte nur mit Einschränkung zu vergleichen sind, da sich die Frage zu Geldzahlungen zwischen den Wellen mehrfach geändert hat (siehe Abschnitt 14.2 Datengrundlage): In der Vergangenheit wurde nicht präzise zwischen Kostenerstattungen und Zugewinnzahlungen unterschieden, da gleichzeitig nach Honoraren und pauschalierten Aufwandsentschädigungen gefragt wurde. Honorare sind zugewinnorientierte Zahlungen, während sich bei pauschalierten Aufwandsentschädigungen Kostenerstattungen und Zugewinnzahlungen mischen können. Im Jahr 2014 wurde zwischen Kostenerstattungen und Zugewinnzahlungen unterschieden - und die freiwillig Engagierten trennen ebenfalls sehr deutlich zwischen Kostenerstattung und finanziellem Zugewinn. Im Jahr 2014 haben 9,9 Prozent der freiwillig Engagierten (zugewinnorientierte) Geldzahlungen erhalten, während 6,9 Prozent der freiwillig Engagierten Kostenerstattungen in Anspruch genommen haben (Abbildung 14-17). Ein großer Teil jener freiwillig Engagierten, die Gebrauch von einer Kostenerstattung machen, hat angegeben, keine zusätzlichen Geldzahlungen zu erhalten. Nur ein kleiner Teil (2,3 Prozent der freiwillig Engagierten) macht von der Kostenerstattung Gebrauch und erhält zusätzlich Geldzahlungen. Betrachtet man Kostenerstattungen und Zugewinnzahlungen gemeinsam, so ergibt sich ein Anteil von 14,5 Prozent der freiwillig Engagierten, die Kostenerstattungen oder Geldzahlungen oder beides erhalten haben. Auch wenn man diesen Wert heranzieht, ist im Zeitvergleich kein Trend zur Monetarisierung festzumachen.

Die Anpassungen in der Abfrage könnten teilweise die für 2014 beobachtete Abnahme an Geldzahlungen erklären. Aber auch wenn ein gewisser Befragungseffekt dergestalt möglich ist, dass die präzisierte Abfrage 2014 zu geringeren Anteilen an Geldzahlungen führt als die Abfrage mit mehreren Beispielen, die 1999 und 2009 eingesetzt wurde, ist eindeutig festzuhalten, dass eine Zunahme an Geldzahlungen zwischen 2009 und 2014 auszuschließen ist.

\section{Abbildung 14-12: Anteile der Engagierten, die Geldzahlungen für die freiwillige Tätigkeit erhalten, im Zeitvergleich}

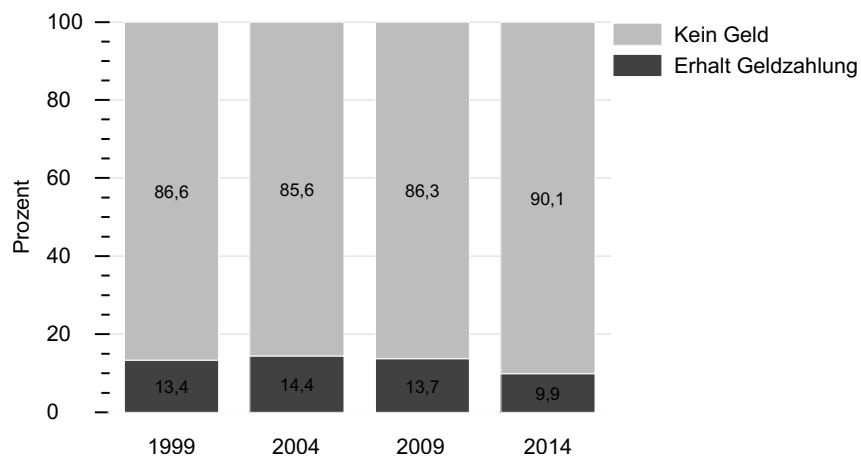

Quelle: FWS, gewichtet, eigene Berechnungen (DZA). Basis: Alle Engagierten. FWS 1999 ( $n=4.671$ ), FWS 2004 $(n=5.137)$, FWS $2009(n=7.001)$, FWS $2014(n=12.417)$. Die Frage zur Vergütung ist zwischen den Wellen mehrfach geändert worden, deswegen sind die Anteile nicht uneingeschränkt vergleichbar. 
Diese abnehmende Tendenz ist prinzipiell sowohl für Frauen und Männer als auch in allen Altersund Bildungsgruppen zu beobachten (Abbildung 14-13). Gleichzeitig gleichen sich die Anteile von Frauen und Männern, die Geld für ihre freiwillige Tätigkeit erhalten, etwas an: Die Differenz hat sich von 6,0 Prozentpunkten auf 2,4 Prozentpunkte reduziert. Insgesamt waren die Anteile von Geldzahlungen darüber hinaus auch in den Jahren 2004 und 2009 gering (14,4 Prozent in 2004 sowie 13,7 Prozent in 2009), aber trotzdem noch höher als im Jahr 2014. Diese Ergebnisse zeigen, dass die verstärkte Debatte über die Monetarisierung nicht durch einen höheren Anteil von Engagierten, die Geldzahlungen für ihre freiwillige Tätigkeiten erhalten, begleitet wird. Dieser Befund zeigt sich trotz großzügigerer steuerlicher Rahmenbedingungen: So hat der Deutsche Bundestag im Juli 2007 unter dem Leitsatz ,Hilfen für Helfer' das, Gesetz zur weiteren Stärkung des bürgerschaftlichen Engagements' (Gesetz zur weiteren Stärkung des bürgerschaftlichen Engagements vom 10. Oktober 2007. Bundesgesetzblatt Jahrgang 2007 Teil I Nr. 50, ausgegeben zu Bonn am 15. Oktober 2007) verabschiedet. Das Gesetz sieht neben einer großzügigeren und transparenten Regelung des Spendenrechts vor, dass Einnahmen aus den in $₫ 3$ Abs. 26 Einkommensteuergesetz (EStG) genannten Tätigkeitsbereichen (z. B. Übungsleiter, Erzieher etc.) bis zu einer Höhe von jährlich 2.400 Euro steuerfrei sind. Für die übrigen Tätigkeitsbereiche (z. B. Platzwarte, Schiedsrichter etc.) kann der Steuerfreibetrag (720 Euro) nach $₫ 3$ Abs. 26a EStG in Anspruch genommen werden.

Möglicherweise wurde der in der Vergangenheit konstatierte Trend zur Monetarisierung jedoch weniger durch Geldzahlungen als vielmehr durch ,Zahlungen' in Form von Sachzuwendungen bedingt. Denn in der Vergangenheit wurde der Monetarisierungstrend nicht allein an Geldzahlungen bemessen, wie ein Blick auf die Ergebnisse des letzten Berichts zum Freiwilligensurvey zeigt: „Der Anteil der Tätigkeiten, für die die Engagierten eine Vergütung erhielten, stieg von 18 Prozent im Jahr 1999 auf 23 Prozent im Jahr 2009 [...]. Das bedeutet, dass der materielle Aspekt des freiwilligen Engagements zwischen 1999 und 2009 an Bedeutung gewonnen hat. Die größte Steigerung gab es bei den Sachzuwendungen (z. B. Fahrscheine, private Nutzung von Gruppenräumen oder Ausstattungsmitteln)“ (Gensicke 2015: 233).

Abbildung 14-13: Anteile der Engagierten, die Geldzahlungen für die freiwillige Tätigkeit erhalten, im Zeitvergleich, a) nach Geschlecht, b) nach Alter und c) nach Bildung

a) nach Geschlecht

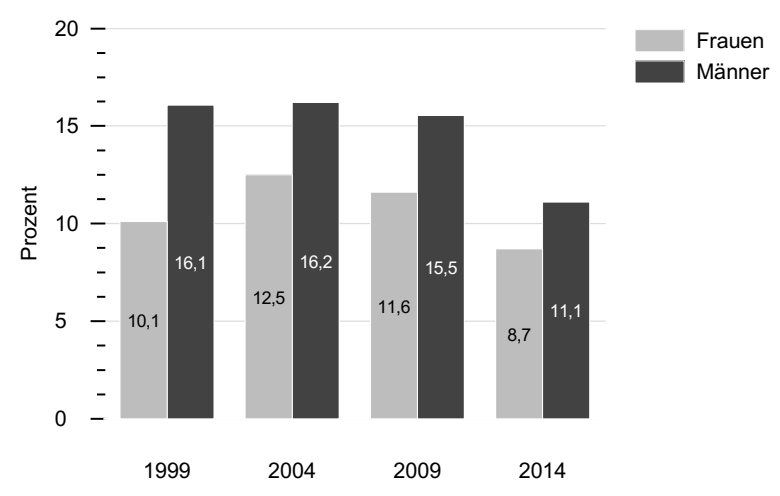




\section{b) nach Alter}

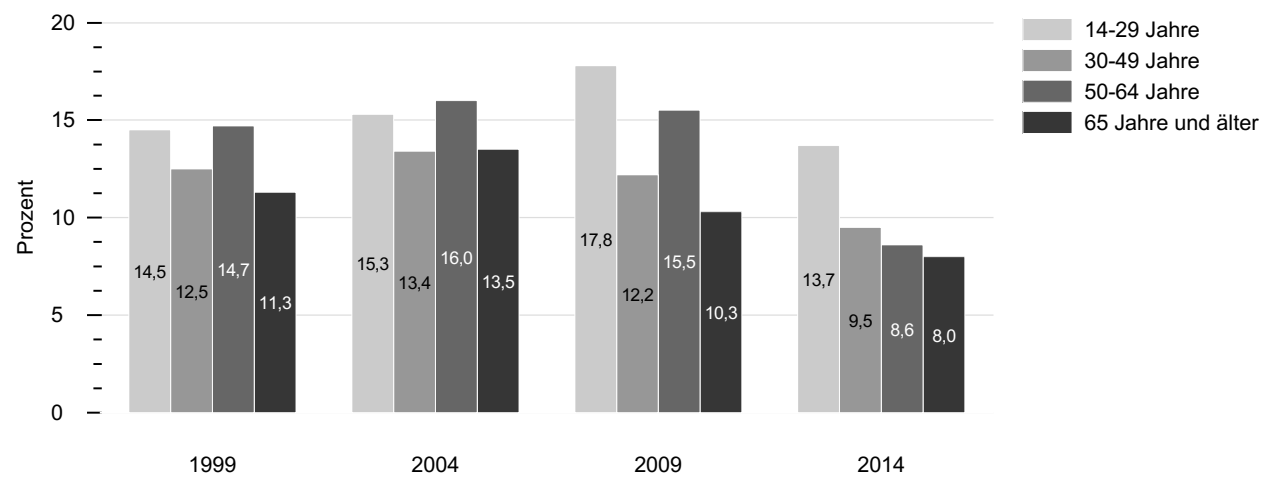

\section{c) nach Bildung}

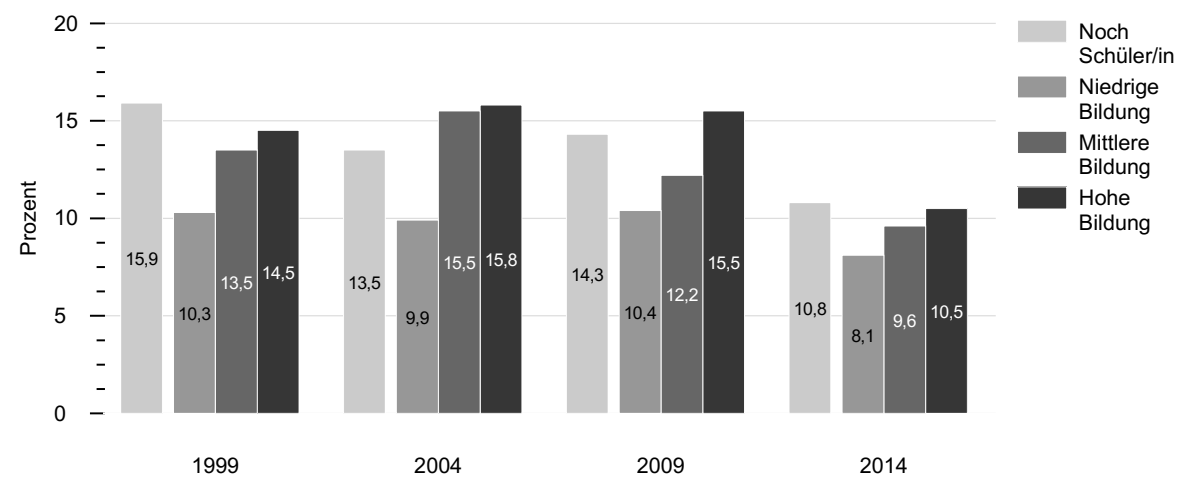

Quelle: FWS, gewichtet, eigene Berechnungen (DZA). Nach Geschlecht: FWS 1999, ( $n=4.671)$, FWS 2004 ( $n=5.137)$, FWS 2009 ( $n=7.001)$, FWS $2014(n=12.417)$, nach Alter: FWS $1999(n=4.666)$, FWS $2004(n=5.127)$, FWS 2009 ( $n=6.991)$, FWS $2014(n=12.417)$, nach Bildung: FWS $1999(n=4.651)$, FWS $2004(n=5.133)$, FWS $2009(n=6.991)$, FWS 2014 ( $n=12.414$ ).

Die Frage zur Vergütung ist zwischen den Wellen mehrfach geändert worden, deswegen sind die Anteile nicht uneingeschränkt vergleichbar. 
In Abbildung 14-14 sehen wir die Entwicklung von Sachzuwendungen seit 1999. Der Anteil der Personen, die Sachzuwendungen erhalten, hat im Zeitraum von fünfzehn Jahren deutlich zugenommen. Im Jahr 1999 betrug der Anteil 5,6 Prozent, das heißt der Anteil der Engagierten, die Sachzuwendungen erhalten haben, ist um neun Prozentpunkte gestiegen und hat sich somit mehr als verdoppelt. Dieser Anstieg geht zu großen Teilen auf Veränderungen in der Altersgruppe der 14- bis 29-Jährigen zurück.

\section{Abbildung 14-14: Anteile der Engagierten, die Sachzuwendungen für die freiwillige Tätigkeit erhalten, im Zeitvergleich}

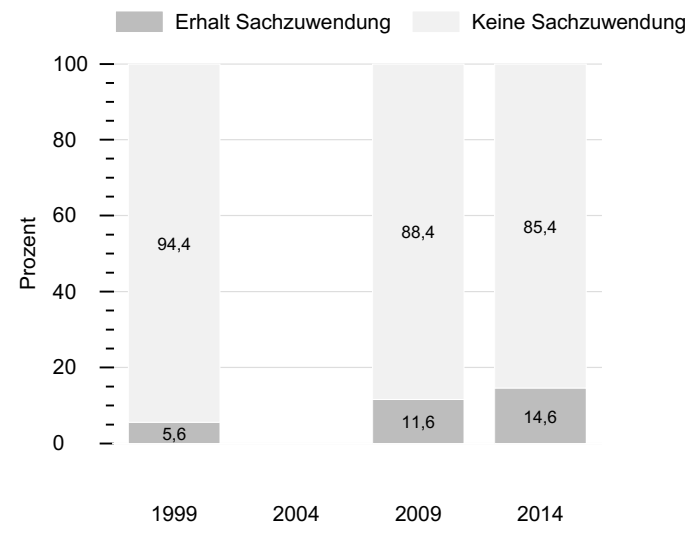

Quelle: FWS, gewichtet, eigene Berechnungen (DZA). FWS $1999(n=4.671)$, FWS $2009(n=7.001)$, FWS 2014 ( $n=12.415)$.

Im Jahr 2004 wurden Sachzuwendungen nicht erhoben.

Abbildung 14-15: Anteile der Engagierten, die Sachzuwendungen für die freiwillige Tätigkeit erhalten, im Zeitvergleich, a) nach Geschlecht, b) nach Alter und c) nach Bildung

a) nach Geschlecht

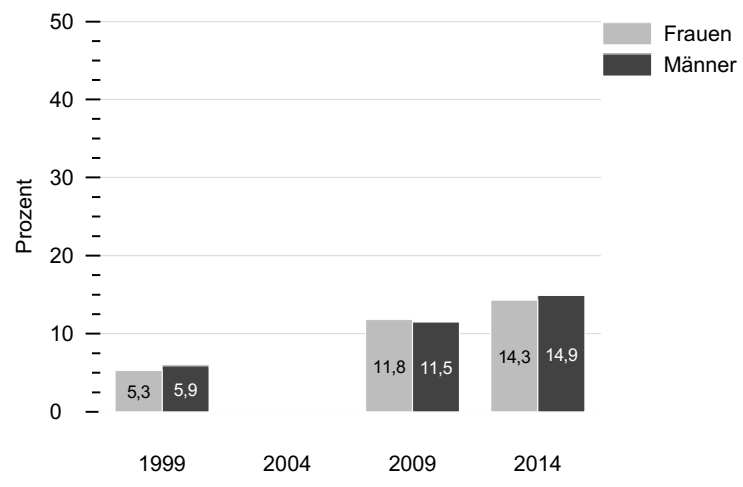




\section{b) nach Alter}

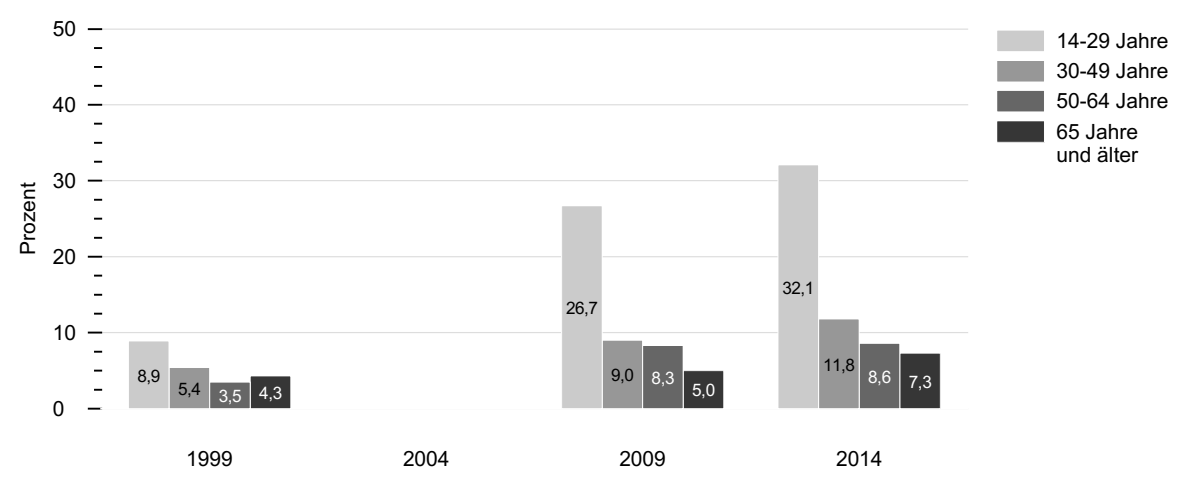

c) nach Bildung

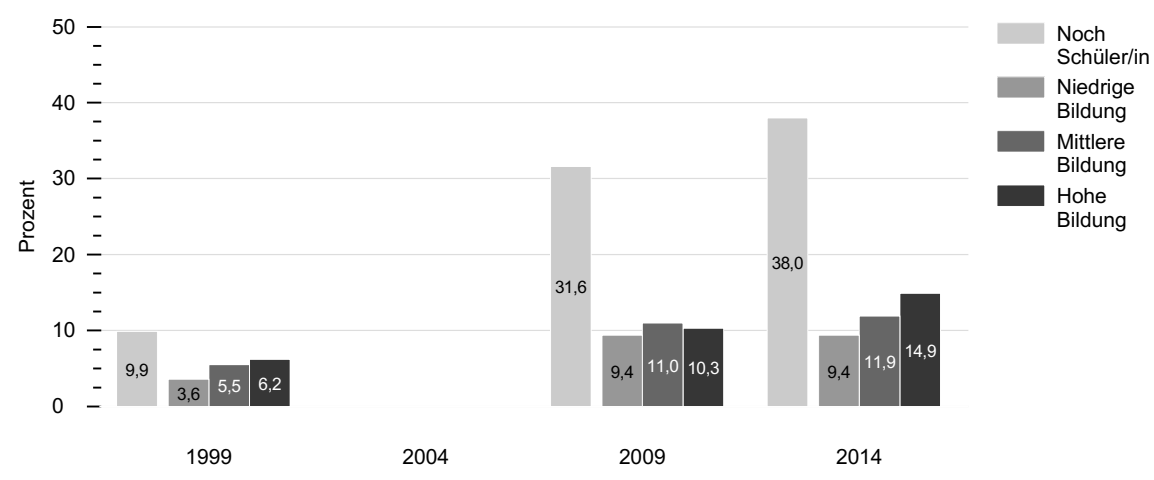

Quelle: FWS, gewichtet, eigene Berechungen (DZA). Nach Geschlecht: FWS 1999 ( $n=4.671)$, FWS 2009 ( $n=7.001$ ), FWS 2014 ( $n=12.415)$, nach Alter: FWS $1999(n=4.666)$, FWS $2009(n=6.991)$, FWS 2014 ( $n=12.415)$, nach Bildung: FWS 1999 ( $n=4.651)$, FWS 2009 ( $n=6.991)$, FWS $2014(n=12.412)$.

Im Jahr 2004 wurden Sachzuwendungen nicht erhoben.

Wir sehen, dass der Anteil von Personen, die Sachzuwendungen erhalten, zwischen 1999 und 2014 in der Gruppe der Schülerinnen und Schüler besonders stark gestiegen ist (von 9,9 Prozent in 1999 auf 38,0 Prozent in 2014; Abbildung 14-15c). Der Anstieg fand auch schon zwischen 1999 und 2009 statt (von 9,9 auf 31,6 Prozent) und hat sich bis zum Jahr 2014 fortgesetzt (es gibt keine Information $\mathrm{zu}$ Sachzuwendungen für das Jahr 2004). Eine mögliche Interpretation für diese starke Entwicklung könnte sein, dass Institutionen zunehmend Sachzuwendungen als Anreiz nutzen, mehr junge Menschen für das freiwillige Engagement zu gewinnen. 


\subsection{Kosten und Möglichkeiten der Kostenerstattung}

Durch freiwillige Tätigkeiten können den Engagierten Kosten entstehen, wenn sie etwa mit privaten Mitteln bestimmte Auslagen oder Anschaffungen übernehmen. Dazu gehören etwa Fahrtkosten, um zu einem Treffen oder einem Arbeitsort zu fahren, möglicherweise auch Reisekosten für Übernachtung und Anfahrt, zudem vielleicht Kosten für Telefon oder Internet beziehungsweise Porto, und auch Sachkosten für Materialien wie Noten oder Ausrüstungsgegenstände wie Musikinstrumente oder Sicherheitskleidung. Je nachdem, für welche Organisation oder Einrichtung man sich engagiert und wie diese Organisation oder Einrichtung finanziell aufgestellt ist, gibt es die Möglichkeit, sich anfallende Kosten erstatten zu lassen.

Finanzielle Kosten werden oft als eine $\mathrm{Zu}$ gangsbarriere für freiwilliges Engagement betrachtet. Die Möglichkeit Kosten erstattet $\mathrm{zu}$ bekommen soll diese Barriere reduzieren. Bei voller Kostenerstattung entstehen insgesamt keine finanziellen Nachteile für die Engagierten. Das heißt, auch Personen mit geringem Einkommen können es sich unter dieser Bedingung leisten, sich zu engagieren und gesellschaftlich teilzuhaben. Die einzigen verbleibenden Barrieren bestehen möglicherweise noch darin, dass bestimmte Beträge zunächst ausgelegt werden müssen oder der bürokratische Aufwand hoch ist, diese nachträglich erstatten zu lassen. Hier sind Personen mit größeren ökonomischen Ressourcen und einem höheren Bildungsniveau im Vorteil, weil sie einen größeren Handlungsspielraum haben. In den Fällen, in denen Engagierte sich ihren finanziellen Aufwand nicht entschädigen lassen, leisten sie neben ihrer Zeitspende noch eine zusätzliche indirekte Geldspende. Es ist anzunehmen, dass indirekte Geldspenden ebenso wie direkte Geldspenden (siehe Kapitel 7) vom Einkommens- und auch vom Bildungsniveau der Engagierten abhängen und häufiger von Personen mit höheren Ressourcen geleistet werden.

In diesem Abschnitt untersuchen wir, wie weit Kosten im freiwilligen Engagement verbreitet sind. Darüber hinaus untersuchen wir die Möglichkeit der Kostenerstattung und wie oft davon Gebrauch gemacht wird. Wir untersuchen die Frage der Kosten allerdings nicht im Zeitvergleich, weil eine direkte Erhebung der entstehenden Kosten im Deutschen Freiwilligensurvey 2014 erstmalig durchgeführt wurde. So ist es möglich, direkt zu berichten, für welche Gruppen von Engagierten überhaupt Kosten durch ihre freiwilligen Tätigkeiten entstehen.

\subsubsection{Kosten im freiwilligen Engagement 2014}

In Abbildung 14-16a sehen wir, dass knapp der Hälfte der engagierten Personen Kosten durch ihr freiwilliges Engagement entstehen (46,3 Prozent). Es gibt Geschlechter-, Altersgruppen- und Bildungsunterschiede bei dem Anteil von Engagierten, denen Kosten im freiwilligen Engagement entstehen (Abbildung 14-16a und b). So entstehen bei engagierten Männern im Durchschnitt öfter Kosten (50,0 Prozent) als bei engagierten Frauen (42,5 Prozent). In der Altersgruppe der 50- bis 64-Jährigen entstehen mit 51,3 Prozent am häufigsten Kosten durch freiwilliges Engagement. Ähnlich häufig sind Kosten auch bei den 65 -jährigen und älteren Engagierten (mit 48,2 Prozent) sowie den 30- bis 49-Jährigen mit 47,7 Prozent. Nur die jüngste Altersgruppe (14 bis 29 Jahre) berichtet deutlich seltener von ihnen entstehenden Kosten (36,7 Prozent). Am seltensten fallen Kosten für Engagierte an, die noch zur Schule gehen (24,8 Prozent). Gut die Hälfte der hochgebildeten Engagierten (50,7 Prozent) gibt an, dass sie Kosten haben, während dies nur für 40,3 Prozent der Engagierten der untersten Bildungsgruppe gilt. 
Abbildung 14-16: Anteile der Engagierten, die angeben, dass ihnen Kosten durch ihre freiwillige Tätigkeit entstehen, 2014, a) gesamt, nach Geschlecht und nach Alter und b) nach Bildung

a) gesamt, nach Geschlecht und nach Alter

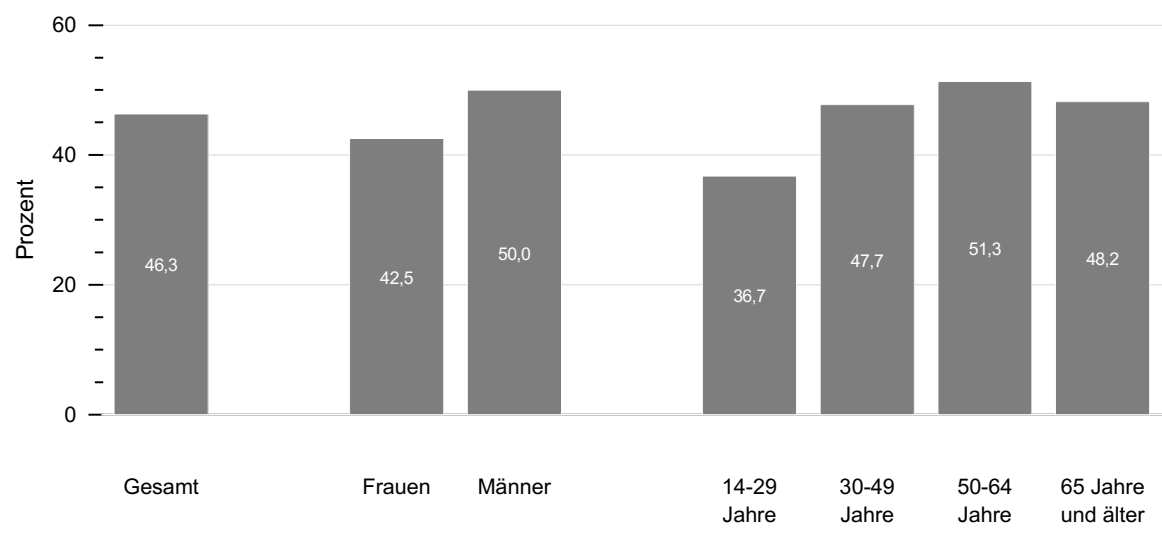

\section{b) nach Bildung}

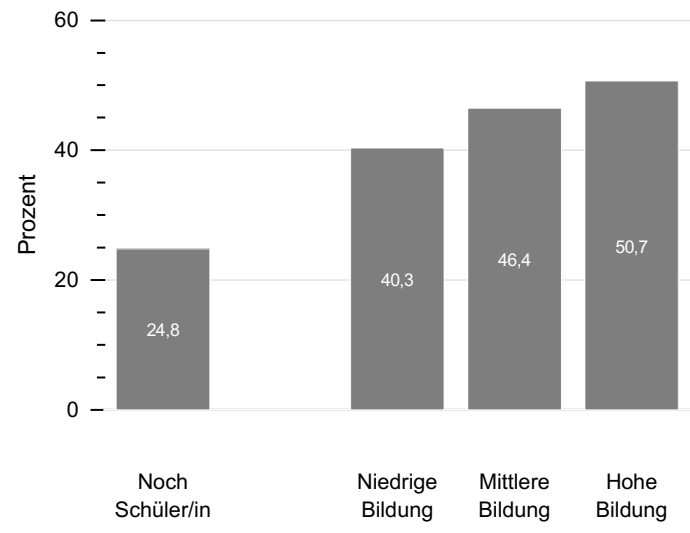

Quelle: FWS 2014, gewichtet, eigene Berechnungen (DZA). Basis: Alle Engagierten. Gesamt, Alter und Geschlecht $(\mathrm{n}=12.228)$, Bildung $(\mathrm{n}=12.225)$. 


\subsubsection{Möglichkeiten der Kosten- erstattung 2014}

Um diese Kosten als Barriere für Engagement interpretieren $\mathrm{zu}$ dürfen, ist es notwendig $\mathrm{zu}$ untersuchen, ob bei den Personen, bei denen die Kosten entstehen, gleichzeitig die Möglichkeit der Kostenerstattung besteht oder ob sie diese Kosten vollständig selbst tragen müssen (Abbildung 14-17). Nur bei 18,6 Prozent der Engagierten ist eine Kostenerstattung möglich (von denen 6,9 Prozent auch davon Gebrauch machen). Ein größerer Anteil von 11,7 Prozent macht davon keinen Gebrauch, diese Gruppe leistet also zusätzlich zur Zeitspende auch noch eine indirekte Geldspende.

Bei Männern ist eine Kostenerstattung öfter möglich als bei Frauen, und Männer nutzen diese Möglichkeit auch öfter (8,1 Prozent versus 5,6 Prozent). Wenn man allerdings nur die Engagierten betrachtet, bei denen überhaupt Kosten entstehen, sind die Geschlechterunterschiede nicht groß. 36,0 Prozent der Frauen und 37,7 Prozent der Männer lassen sich entstandene Kosten erstatten.

Abbildung 14-17: Anteile der Engagierten, die Angaben zur Kostenerstattung machen, 2014, a) gesamt, nach Geschlecht und nach Alter und b) nach Bildung

a) gesamt, nach Geschlecht und nach Alter

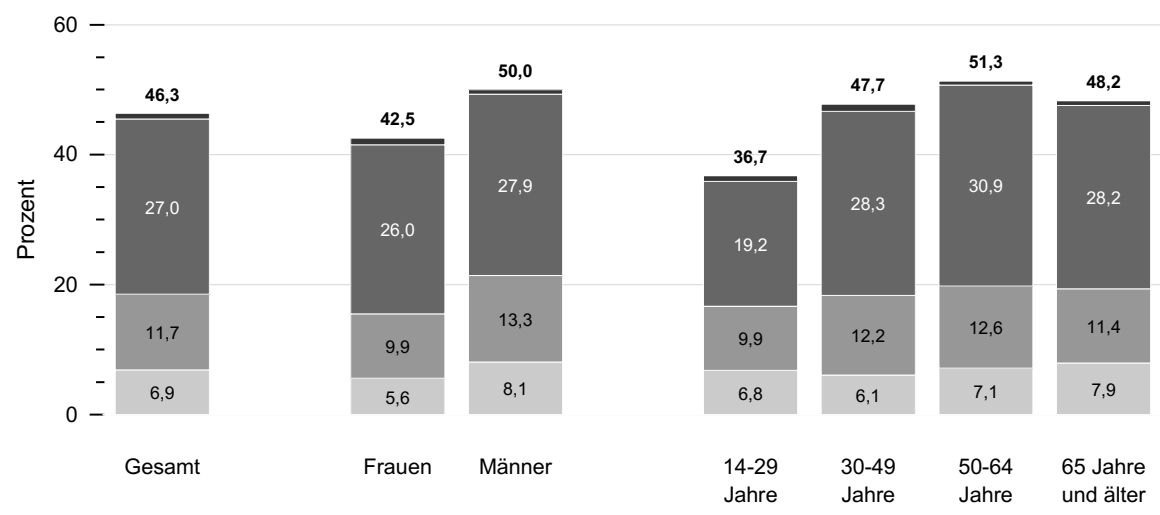




\section{b) nach Bildung}

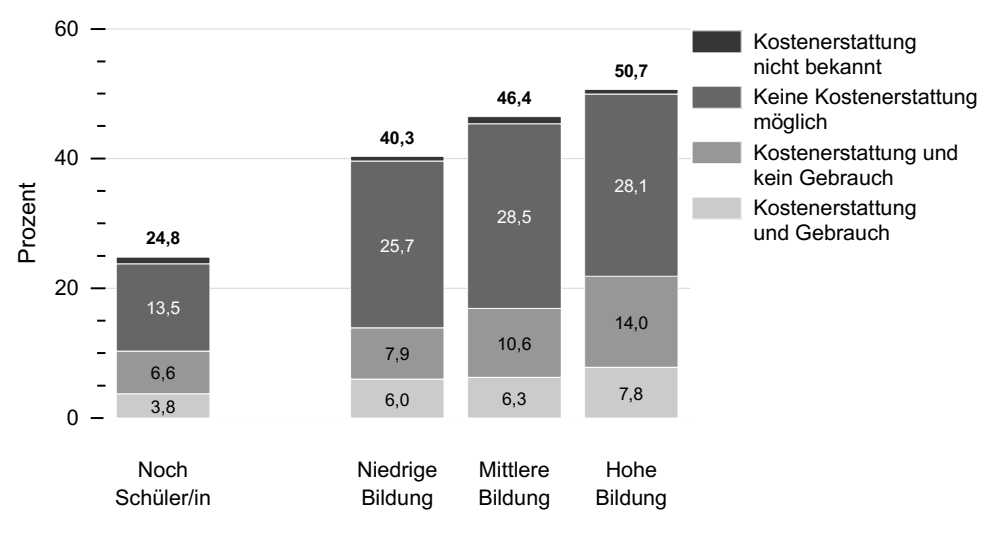

Quelle: FWS 2014, gewichtet, eigene Berechnungen (DZA). Basis: Alle Engagierten. Gesamt, Alter und Geschlecht $(n=12.228)$, Bildung $(n=12.225)$.

Bei Personen mit hoher Bildung ist öfter eine Kostenerstattung möglich (21,8 Prozent) als bei der niedrigen Bildungsgruppe (13,9 Prozent). Hinsichtlich der Inanspruchnahme dieser Möglichkeit sind keine großen Unterschiede zwischen den Bildungsgruppen zu beobachten. Der größte
Unterschied besteht darin, dass Engagierte mit hoher Bildung deutlich häufiger eine indirekte Geldspende leisten, indem sie auf Kostenerstattung verzichten (14,0 Prozent) als Engagierte mit geringer Bildung (7,9 Prozent). 
Abbildung 14-18: Anteile der Engagierten, die angeben, dass ihnen Kosten durch ihre freiwillige Tätigkeit entstehen, 2014, nach Haushaltsnettoeinkommen

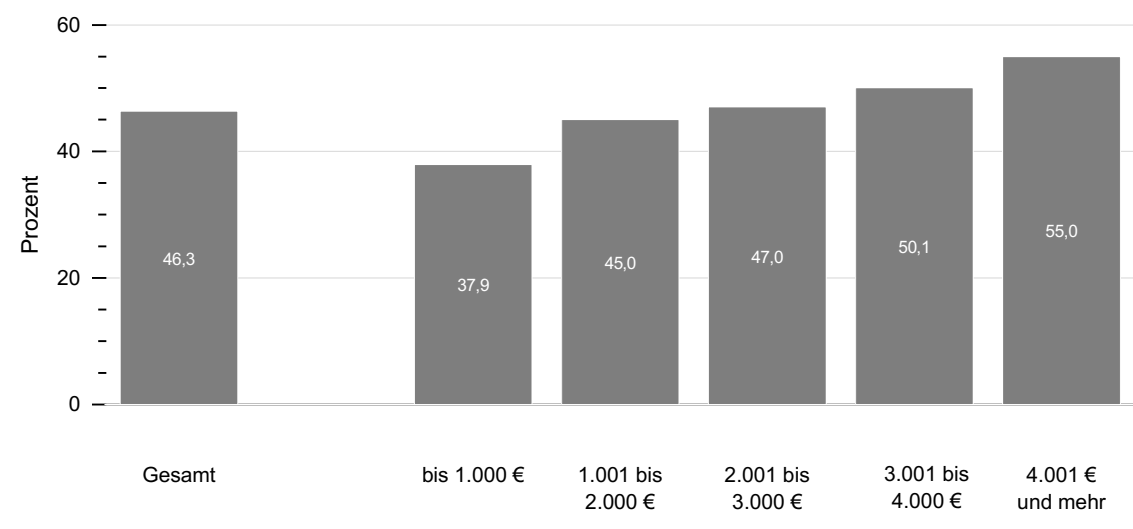

Quelle: FWS 2014, gewichtet, eigene Berechnungen (DZA). Basis: Alle Engagierten mit gültigen Angaben zum Haushaltsnettoeinkommen $(n=10.178)$.

Da Kosten eine finanzielle Barriere darstellen können, sollten Kosten und Kostenerstattung in Verbindung mit dem jeweiligen Einkommen betrachtet werden, das den Engagierten zur Verfügung steht. Es ist festzustellen, dass engagementbedingte Kosten häufiger in höheren Haushaltseinkommensgruppen entstehen (Abbildung 14-18). Mehr als die Hälfte der Engagierten mit einem monatlichen Haushaltseinkommen über 4.000 Euro berichtet, dass ihnen Kosten entstehen (55,0 Prozent), während das nur auf 37,9 Prozent der Engagierten mit einem monatlichen Haushaltseinkommen von 1.000 Euro und weniger zutrifft. Für Engagierte mit höherem Haushaltseinkommen gibt es auch häufiger Erstattungsmöglichkeiten (23,2 Prozent gegen 13,0 Prozent; Abbildung 14-19). Sie machen jedoch davon seltener Gebrauch als Engagierte mit niedrigerem Haushaltseinkommen.

Es zeigt sich aber auch, dass Personen, denen ein niedrigeres Haushaltseinkommen zur Verfügung steht, seltener Kosten entstehen und sie sich diese, wenn möglich, häufiger erstatten lassen. Gebrauch machen davon 6,6 Prozent der Engagierten mit einem Haushaltseinkommen bis 1.000 Euro, keinen Gebrauch davon macht mit 6,4 Prozent eine vergleichbar große Gruppe. Bei den Personen mit einem Haushaltseinkommen von 4.000 Euro und mehr ist das Verhältnis 6,1 Prozent, die die Kostenerstattung nutzen, zu 17,1 Prozent, die darauf verzichten. Dieses Ergebnis zeigt, dass indirekte Geldspenden ebenso wie direkte Geldspenden häufiger von Personen geleistet werden, die in Haushalten mit hohem Einkommen leben.

Da sich jedoch nicht alle Engagierten mit einem Haushaltseinkommen von bis $\mathrm{zu} 1.000$ Euro ihre Kosten - wenn dies überhaupt möglich ist - erstatten lassen, können wir nicht direkt auf größere finanzielle Barrieren für diese Gruppen schließen. Allerdings besteht hier zusätzlich ein Selektionsproblem. Wir können an dieser Stelle nur auf die Rolle von Kosten und Kostenerstattung als finanzielle Barriere für die Personen, die schon engagiert sind, hinweisen. Wir können nicht empirisch überprüfen, inwiefern die Kosten ein entscheidender Faktor für die Nicht-Engagierten sind, sich aufgrund der zu erwartenden finanziellen Belastung nicht zu engagieren. Allerdings zeigt sich, dass die Wahrscheinlichkeit, sich zu engagieren mit geringerem Einkommen ohnehin kleiner ist (siehe Kapitel 16). Wir können jedoch direkt aus der empirischen Analyse der Engagierten mit einem Haushaltseinkom- 
men von bis zu 1.000 Euro ablesen, dass auch hier noch Handlungsbedarf besteht: Knapp ein Viertel kann sich die Kosten gar nicht erstatten lassen. Hier besteht also weiterhin eine finanzielle Barriere, die von den zivilgesellschaftlichen Organisationen noch abgebaut werden kann.
Zwar ist der Anteil mit 30,8 Prozent bei den Engagierten mit höchstem Einkommen noch größer, allerdings erscheint der Handlungsbedarf hier deshalb weniger dringend, weil bei Einkommen in dieser Höhe Kosten für Engagement nicht so stark ins Gewicht fallen dürften.

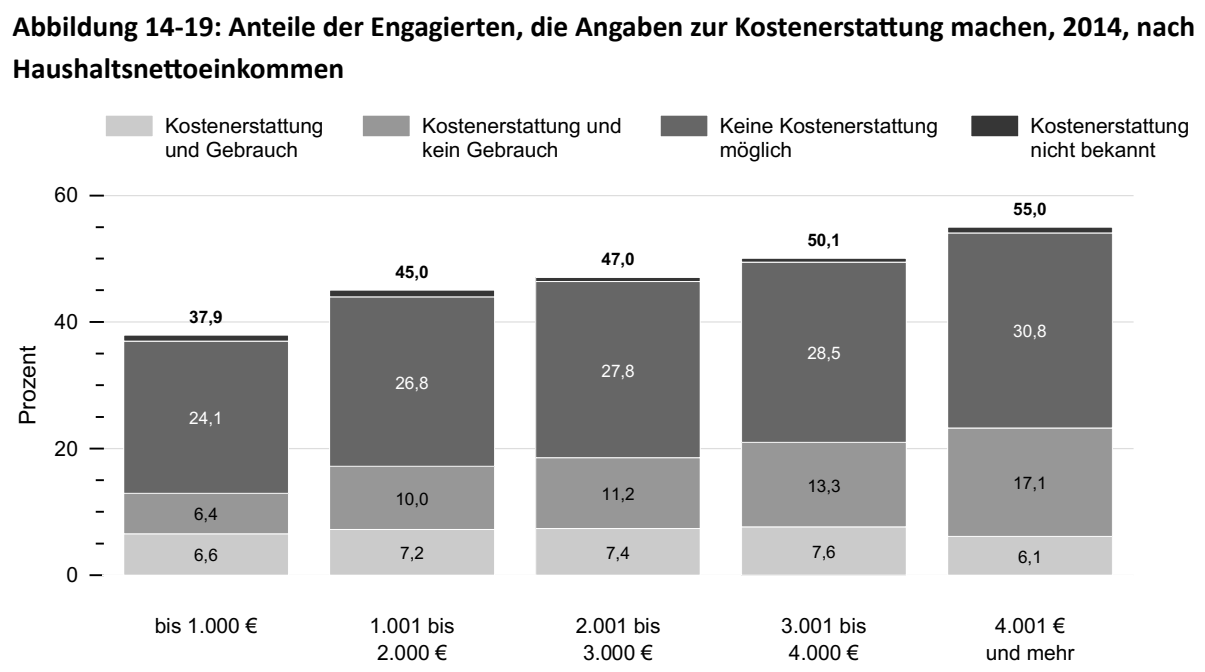

Quelle: FWS 2014, gewichtet, eigene Berechnungen (DZA). Basis: Alle Engagierten mit gültigen Angaben zum Haushaltsnettoeinkommen $(n=10.178)$. 


\subsubsection{Kosten in den gesellschaft- lichen Engagementbereichen}

Es ist anzunehmen, dass die Möglichkeiten zur Kostenerstattung tatsächlich von den finanziellen Möglichkeiten der jeweiligen Organisationen oder Einrichtungen abhängen und weniger von den individuellen Eigenschaften der Engagierten. Ein Beispiel: Engagiert sich jemand alleine und individuell, zum Beispiel als unabhängiger Online-Volunteer, hat sie oder er auch keine Organisation im Rücken, die anfallende Kosten für den Internetauftritt oder den Computer erstatten könnte. Vereine hingegen nehmen Mitgliedschaftsbeiträge ein und können so auch bei- spielsweise Übungsleiterpauschalen finanzieren (siehe Kapitel 8).

Die Analyse der Engagementbereiche kann zeigen, wo finanzielle Barrieren für das Engagement bestehen (Abbildung 14-20). Die Bereiche, in denen am häufigsten Kosten für eine Tätigkeit entstehen, sind: Umwelt (56,5 Prozent), Kultur und Musik (54,1 Prozent), Politik (52,3 Prozent) sowie Sport und Bewegung (51,3 Prozent). Auch in der Jugendarbeit fallen überdurchschnittlich häufig Kosten an (49,0 Prozent). Dagegen entstehen am seltensten Kosten in den Bereichen Freizeit und Geselligkeit (35,9 Prozent) sowie Schule und Kindergarten (36,1 Prozent).

\section{Abbildung 14-20: Anteile der Engagierten, die angeben, dass ihnen Kosten durch ihre freiwillige Tätigkeit entstehen, 2014, nach Bereichen}

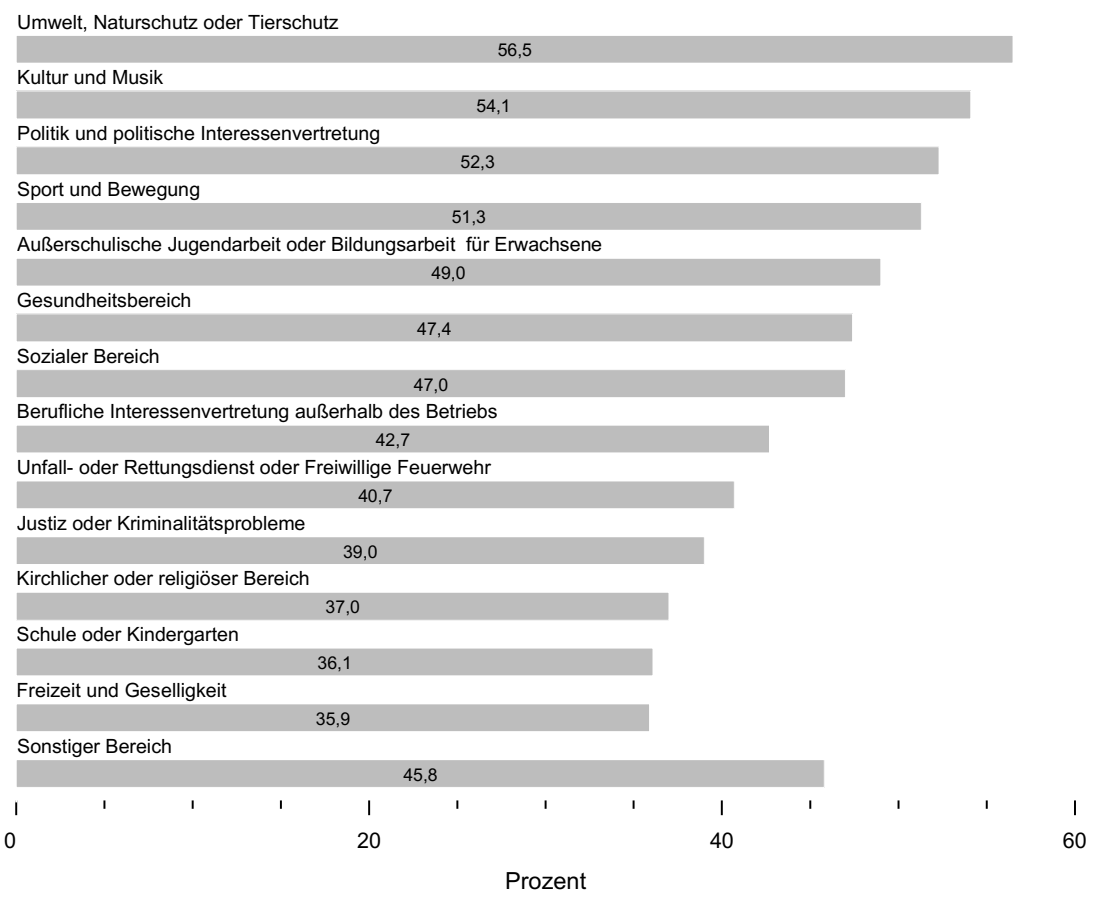

Quelle: FWS 2014, gewichtet, eigene Berechnungen (DZA). Basis: Alle Engagierten ( $n=12.327$. 
Die Bereiche, in denen am häufigsten von entstehenden Kosten berichtet wird, korrespondieren nicht mit den Bereichen, in denen die Engagierten am häufigsten Geldzahlungen oder Sachzuwendungen erhalten. Zum Beispiel gibt es im Bereich Umwelt, in dem am häufigsten Kosten entstehen, sehr selten Geldzahlungen (nur für 6,6 Prozent, Abbildung 14-5) und auch Sachzuwendungen werden selten bereitgestellt (nur für 11,7 Prozent, Abbildung 14-7). In diesem Bereich er- bringen die Engagierten neben ihrer Zeitspende auch am häufigsten indirekte Geldspenden, weil sie die Kosten für ihr Engagement überwiegend selbst tragen.

In Abbildung 14-21, werden die Geschlechterunterschiede in den verschiedenen Bereichen dargestellt. In den meisten Bereichen berichten Männer häufiger als Frauen, dass ihnen aus ihrer freiwilligen Tätigkeit Kosten entstehen.

\section{Abbildung 14-21: Anteile der Engagierten, die angeben, dass ihnen Kosten durch ihre freiwillige Tätigkeit entstehen, 2014, nach Bereichen sowie nach Geschlecht}

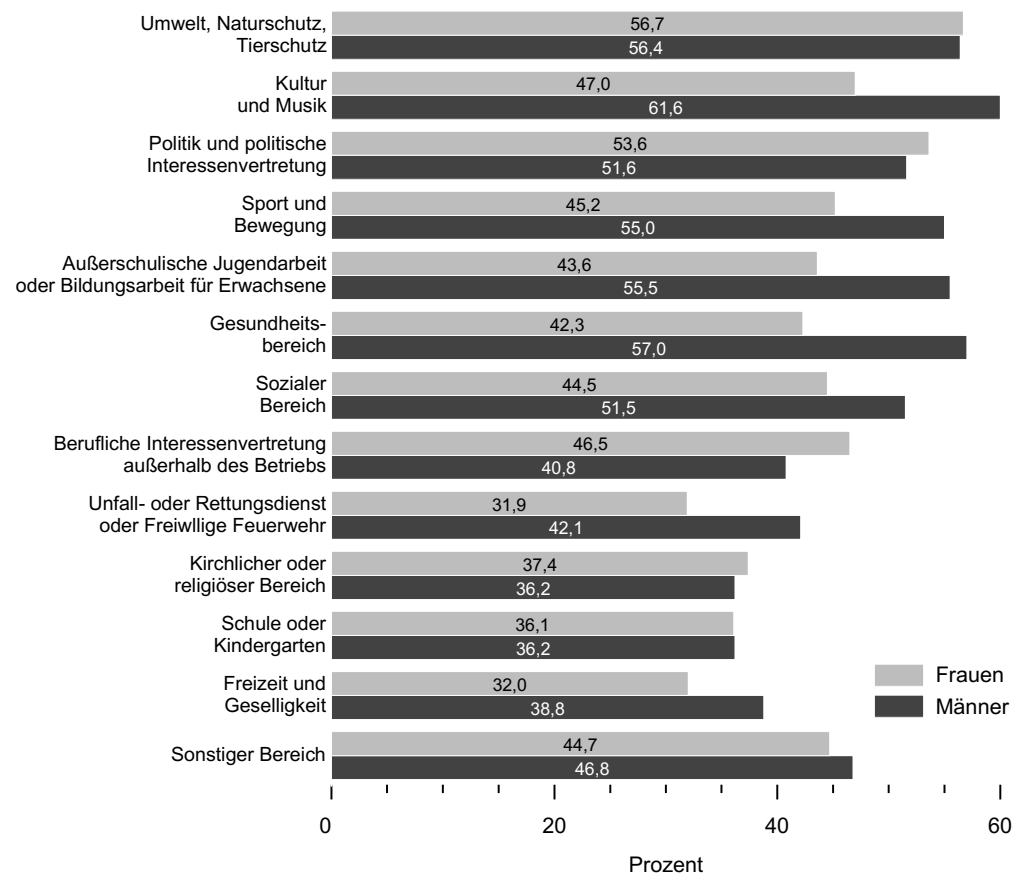

Quelle: FWS 2014, gewichtet, eigene Berechnungen (DZA). Basis: Alle Engagierten $(n=12.253$ ).

Für den Bereich ,Justiz oder Kriminalitätsprobleme' werden keine Ergebnisbalken nach Geschlecht dargestellt, da im Datensatz jeweils weniger als 50 Fälle engagierter Frauen und Männer in diesem Bereich enthalten sind. 


\subsection{Fazit}

Die Anteile von Engagierten, die Geldzahlungen oder Sachzuwendungen für ihre freiwillige Tätigkeit erhalten, sind niedrig. Nur 9,9 Prozent der Engagierten erhalten Geldzahlungen und die Beträge sind in der Regel niedrig (meistens unter 50 Euro). Darüber hinaus ist auch der Anteil der Engagierten, die berichten, dass für sie Sachzuwendungen bereitgestellt werden, nicht hoch (14,6 Prozent). Einzige Ausnahme ist die Gruppe der Schülerinnen und Schüler, die für ihr Engagement sehr häufig (38,0 Prozent) Sachzuwendungen erhält. Die Geschlechterunterschiede sind größer bei Geldzahlungen $(11,1$ Prozent der engagierten Männer und 8,7 Prozent der engagierten Frauen erhalten Geldzahlungen) als bei Sachzuwendungen, bei denen die Anteile für Frauen (14,3 Prozent) und Männer (14,9 Prozent) ähnlicher sind.

Geldzahlungen und Sachzuwendungen variieren je nach gesellschaftlichem Engagementbereich. Darüber hinaus werden Geld- und Sachzuwendungen selten kombiniert. So erhalten die Engagierten in dem Bereich Justiz oder Kriminalitätsprobleme sehr häufig Geldzahlungen und sehr selten Sachzuwendungen, während im Bereich Kultur und Musik das Gegenteil zu beobachten ist. Dieses Ergebnis zeigt, dass die Vergütungsform sehr bereichsspezifisch ist.

Im Zeitvergleich beobachten wir, dass Geldzahlungen für freiwillige Tätigkeiten seit 1999 nicht zugenommen haben. Engagierte erhalten 2014 sogar zu etwas geringeren Anteilen Geldzahlungen für ihre freiwillige Tätigkeiten als in früheren Erhebungsjahren, was unter anderem daran liegen könnte, dass im Zeitvergleich seltener ausgeübtes Engagement stärker angestiegen ist als häufiger ausgeübtes Engagement (siehe Kapitel 12) und seltener ausgeübtes Engagement in der Regel auch seltener vergütet wird als häufig ausgeübtes Engagement. Auf Basis dieser Befunde kommen wir zu dem Schluss, dass sich empirisch für das freiwillige Engagement insgesamt kein Trend zur Monetarisierung belegen lässt. Diese Interpretation ist möglich, obwohl sich die Frage zu Geldzahlungen zwischen den Wellen jeweils verändert hat. So wurde in früheren Wellen separat nach pauschalierten Aufwandsentschädigungen, Honoraren und geringfügiger Bezahlung gefragt (2004) beziehungsweise nach pauschalierten Aufwandsentschädigungen, Honoraren, geringfügiger Bezahlung und Sachzuwendungen (2009), was zu einer höheren Zahl von Nennungen führen kann als die präzisierte Erhebung 2014, in der nach Kostenerstattung einerseits und Geldzahlungen im Sinne eines finanziellen Zugewinnes andererseits gefragt wurde. Selbst wenn man Kostenerstattungen und zugewinnorientierte Geldzahlungen kombiniert, ist im Zeitvergleich kein Trend zur Monetarisierung festzustellen.

Bei freiwillig engagierten Schülerinnen und Schüler hat der Anteil derjenigen, für die Sachzuwendungen bereitgestellt werden, stark zugenommen. Während im Jahr 1999 nur 10,8 Prozent der Schülerinnen und Schüler Sachzuwendungen erhalten haben, beträgt dieser Anteil im Jahr 2014 weit überdurchschnittliche 38,0 Prozent. Hier stellt sich die Frage, ob dieser Trend das Resultat eines höheren Wettbewerbs der Organisationen ist, Jüngere für freiwillige Arbeiten und Aufgaben zu mobilisieren. Es ist zu überlegen, ob der Versuch, jüngeren Engagierten mit Sachzuwendungen Anreize zu setzen, der Idee des freiwilligen Engagements widerspricht. Junge Engagierte könnten sich dadurch zu sehr an Leistungen orientieren und die intrinsische Motivation für das Engagement könnte sich verringern. Um dies zu überprüfen, müssten jedoch verschiedene Arten von Sachzuwendungen in den Blick genommen werden.

Darüber hinaus berichtet fast die Hälfte der Engagierten (46,3 Prozent), dass ihnen im Rahmen ihrer freiwilligen Tätigkeit Kosten entstehen. Hier stellt sich die Frage, ob diese Kosten eine finanzielle Last für die Engagierten sind. Die Ergebnisse zeigen, dass die Möglichkeiten für die Erstattung der entstandenen Kosten im freiwilligen Engagement nicht sehr weit verbreitet sind. 
Und selbst dort, wo Erstattungsmöglichkeiten bestehen, nehmen nicht alle Engagierten diese auch in Anspruch. Abhängig vom Bildungsabschluss und Einkommensniveau entscheiden sich Engagierte mit höheren individuellen Ressourcen sogar deutlich häufiger, die Kosten selbst zu tragen - wahrscheinlich, weil sie es sich eher leisten können. Sie spenden somit indirekt auch Geld an die Organisation oder Institution, für die sie sich freiwillig engagieren.

Wie Geldzahlungen im freiwilligen Engagement $\mathrm{zu}$ bewerten sind, wird sehr kontrovers diskutiert. Einige argumentieren zum Beispiel, Geldleistungen könnten schaden, weil sie die Freiheit und Selbstbestimmung der Engagierten beschneiden und demotivierend wirken. Andere kritisieren die Bezahlung freiwilliger Tätigkeiten grundsätzlich deshalb, weil es sich hierbei um prekäre Beschäftigung handelt (Jakob 2013). Dies ist der Fall, wenn schlecht bezahlte Ehrenamtliche lediglich eingesetzt werden, um Aufgaben zu übernehmen, die eigentlich von Seiten des Staates geleistet beziehungsweise finanziert werden müssten, etwa im Bereich der Bildung oder der Altenpflege (Pinl 2015). Befürworterinnen und Befürworter von Geldleistungen betonen hingegen den Aspekt der Ermöglichung von Engagement und der Mobilisierung zum Engagement durch Geld- oder Sachzuwendungen als Anreiz. Die Ergebnisse des Freiwilligensurveys 2014 zeigen allerdings, dass die Engagementquoten seit 1999 stark zugenommen haben (siehe Kapitel 3), obwohl es keine Evidenz für einen Monetarisierungstrend gibt. Dies lässt vermuten, dass Geldzahlungen nicht den entscheidenden Faktor ausmachen, der das zunehmende Engagement erklären kann. Grundsätzlich sollten Geldzahlungen deshalb auch nicht als Mittel zur Mobilisierung neuer Engagierter eingesetzt werden, auch wenn sie in einigen Engagementbereichen ihre Berechtigung haben mögen. Andere Motive zeigen sich relevanter für freiwilliges Engagement. Die Ergebnisse des Kapitels 15 zu Motiven für Engagement bestätigen diese Vermutung. Die meisten Engagierten berichten als Hauptmotiv, Spaß zu haben und mit anderen Menschen zusammenkommen zu wollen, und die wenigsten berichten als Motiv, etwas dazu verdienen zu wollen. Das bedeutet aber nicht, dass die Relevanz einer Monetarisierungsdebatte für einzelne Arbeitsfelder nicht gegeben sein kann, etwa für soziale Dienstleistungen in der Betreuung und Pflege.

Darüber hinaus bleiben aufgrund der begrenzten finanziellen Ressourcen und des Wunsches von Organisationen, Personen zum freiwilligen Engagement zu animieren, Geldzahlungen ein relevantes Thema auf Organisationsebene. Insbesondere dort, wo häufiger Kosten im Engagement entstehen, sollten die Organisationen und Einrichtungen die Möglichkeiten der Kostenerstattung verbessern, sodass keine finanziellen Barrieren zum Engagement bestehen, gerade für Personen mit wenig individuellen Ressourcen.

\section{Literatur}

Armbrüster, K. (2014). Trilog: Monetarisierung. Ein Beitrag zu rechtlichen Einordnung des Ehrenamtes. Online: http:// www.ehrenamtsbibliothek.de/literatur/pdf_549.pdf (zuletzt abgerufen 16.2.2016).

Ebert, O. (2011). Monetarisierung der Freiwilligenarbeit in Deutschland. In: H. Ammann (Hrsg.) Grenzen-Los! Fokus Gemeinde. Freiwilliges Engagement in Deutschland, Österreich und der Schweiz. Referate der 2. Internationalen Vernetzungskonferenz 2010 in Rüschlikon (S. 273-285). Zürich: Seismo.

Evers, A. (2007). Erwerbsarbeit und Engagement. Beziehungsmuster, Spannungen und Widersprüche. In: Bundesnetzwerk Bürgerschaftliches Engagement (BBE) (Hrsg.) Engagement und Erwerbsarbeit. Dokumentation der Fachtagung am 8. und 9. November 2007 in Berlin (S. 8-13). Berlin: Bundesnetzwerk Bürgerschaftliches Engagement. 
Evers, A. (2006). Wenn Welten durcheinander geraten. Monetarisierung, bezahlte Arbeit und freiwilliges Engagement. In: P. Farago \& H. Ammann (Hrsg.) Monetarisierung der Freiwilligkeit. Referate und Zusammenfassungen der 5. Tagung der Freiwilligenuniversität vom 30. bis 31.5.2005 in Luzern (S. 63-74). Zürich: Seismo.

Frey, B. S., \& Jegen, R. (2001). Motivation Crowding Theory. Journal of Economic Surveys, 15(5), 589-611.

Gensicke, T. (2015). Freiwilliges Engagement in Deutschland: Freiwilligensurvey 2009. Wiesbaden: Springer VS.

Grant, R. W. (2012). Strings Attached: Untangling the Ethics of Incentives. Princeton: Princeton University Press and Russel Sage Foundation.

Jakob, G. (2013). Bezahltes Engagement - Zu den Hintergründen und Risiken einer Monetarisierung bürgerschaftlichen Engagements (eNewsletter Wegweiser Bürgergesellschaft 22/2013). Online: http://www.buergergesellschaft.de/ fileadmin/pdf/gastbeitrag_jakob_131122.pdf (zuletzt abgerufen 16.2.2016).

Jakob, G. (2009). Anerkennungskultur als Ausdruck einer Modernisierung des freiwilligen Engagements. Zeitschrift des vhw - Bundesverband für Wohnen und Stadtentwicklung e.V., 2009(1), 3-6.

Klie, T. (2015). Sieben Thesen zur Monetarisierung (eNewsletter Wegweiser Bürgergesellschaft 04/2015). Online: http://www.buergergesellschaft.de/fileadmin/pdf/gastbeitrag_klie_150311.pdf (zuletzt abgerufen 5.2.2016).

Olk, T., \& Klein, A. (2007). Erwerbsabeit und Engagement. Eine notwendige Diskussion. In: Bundesnetzwerk Bürgerschaftliches Engagement (BBE) (Hrsg.) Engagement und Erwerbsarbeit. Dokumentation der Fachtagung am 8. und 9. November 2007 in Berlin (S. 5-7). Berlin: Bundesnetzwerk Bürgerschaftliches Engagement.

Pinl, C. (2015). Ehrenamt statt Sozialstaat? Kritik der Engagementpolitik. Aus Politik und Zeitgeschichte, 65(14/15), 49-54.

Priller, E., \& Schupp, J. (2011). Soziale und ökonomische Merkmale von Geld- und Blutspendern in Deutschland. DIW Wochenbericht, 78(29), 3-10.

Schumacher, J. (2015). Kooperation von Haupt- und Ehrenamtlichen als Gestaltungsaufgabe. Ein Leitfaden für die Praxis. Auf Grundlage der Ergebnisse der Studie "Kooperation von Haupt- und Ehrenamtlichen in Pflege, Sport und Kultur". Berlin: Bundesministerium für Familie, Senioren, Frauen und Jugend.

Schupp, J. (2012). Die verborgenen Kosten monetärer Anreize. Lohnt sich Motivierung durch Incentivierung? DIW Wochenbericht, 79(6), 20.

Vandamme, R. (2007). Monetarisierung und Bürgerschaftliches Engagement - Plädoyer für eine konsequente Stärkung des Bürgerschaftlichen Engagements! BBE-Newsletter, 24.

Zentrum für Zivilgesellschaftliche Entwicklung (2009). Untersuchung zur Monetarisierung von Ehrenamt und Bürgerschaftlichen Engagement in Baden-Württemberg. Freiburg im Breisgau: Zentrum für Zivilgesellschaftliche Entwicklung.

Open Access Dieses Kapitel wird unter der Creative Commons Namensnennung 4.0 International Lizenz (http://creativecommons.org/licenses/by/4.0/deed.de) veröffentlicht, welche die Nutzung, Vervielfältigung, Bearbeitung, Verbreitung und Wiedergabe in jeglichem Medium und Format erlaubt, sofern Sie den/die ursprünglichen Autor(en) und die Quelle ordnungsgemäß nennen, einen Link zur Creative Commons Lizenz beifügen und angeben, ob Änderungen vorgenommen wurden.

Die in diesem Kapitel enthaltenen Bilder und sonstiges Drittmaterial unterliegen ebenfalls der genannten Creative Commons Lizenz, sofern sich aus der Abbildungslegende nichts anderes ergibt. Sofern das betreffende Material nicht unter der genannten Creative Commons Lizenz steht und die betreffende Handlung nicht nach gesetzlichen Vorschriften erlaubt ist, ist für die oben aufgeführten Weiterverwendungen des Materials die Einwilligung des jeweiligen Rechteinhabers einzuholen. 